\title{
Skp2 Inhibitor AAA-237 Exerts Anti-Tumor Effect on NSCLC by Inducing Mitochondria-Dependent Apoptosis and Senescence
}

Jinyi Liu

Institute of Materia Medica, Chinese Academy of Medical Science and Peking Union Medical College Xiangjin Zheng

Chinese Academy of Medical Science and Peking Union Medical College

\section{Wan Li}

Institute of Materia Medica, Chinese Academy of Medical Science and Peking Union Medical College

\section{Liwen Ren}

Chinese Academy of Medical Science and Peking Union Medical College

\section{Sha Li}

Institute of Materia Medica, Chinese Academy of Medical Science and Peking Union Medical College

\section{Yihui Yang}

Chinese Academy of Medical Science and Peking Union Medical College

\section{Binbin Ge}

Institute of Materia Medica, Chinese Academy of Medical Science and Peking Union Medical College

\section{Guanhua Du}

Institute of Materia Medica, Chinese Academy of Medical Science and Peking Union Medical College Jianyou Shi

University of Electronic Science and Technology of China

Jinhua Wang ( $\square$ wjh@imm.ac.cn )

Insititue of Materia Medica, Chinese Academy of Medical Science \& Peking Union Medical College

\section{Research}

Keywords: AAA-237, Skp2, cell cycle, apoptosis, senescence

Posted Date: July 9th, 2021

DOI: https://doi.org/10.21203/rs.3.rs-656678/v1

License: (c) (i) This work is licensed under a Creative Commons Attribution 4.0 International License.

Read Full License 


\section{Abstract}

Background: Lung cancer is by far the leading cause of cancer death worldwide, and $85 \%$ of patients are non-small cell lung cancer (NSCLC). NSCLC is still very difficult to treat. Skp2 functions as an oncogene and participates in the processes of NSCLC. Here, we disclose a novel Skp2 inhibitor, which is firstly synthesized by our group based on our previous computer virtual screening and biomolecular interaction analysis (Biacore) technology screening.

Methods: To unveil antitumor activity and underlying molecular mechanism of AAA-237, cell proliferation, migration and invasion assays, apoptosis, cell cycle, senescence, and xenograft mouse models were used.

Results: AAA-237 inhibited the proliferation of NSCLC cells. AAA-237 regulated the process of epithelialmesenchymal transition (EMT) by Skp2/Twist axis. Furthermore, AAA-237 induced arresting of cell cycle at G0/G1 phase by regulating the Skp2-Cip/Kip and PI3K/Akt-FOXO1 signaling pathways. More importantly, we found that treatment of high concentration AAA-237 could induce apoptosis of NSCLC cells via the mitochondrial pathway whereas treatment of low concentration AAA-237 for long-term could induce senescence of NSCLC cells by DNA damage. Senolytic drug quercetin could selectively eliminate senescent cells induced by AAA-237. In A549 xenograft nude mice model, AAA-237 dose-dependently inhibited the growth of tumor by inducing apoptosis and senescence.

Conclusions: Taken together, we propose that AAA-237 is a promising therapeutic agent of NSCLC, especially when combined with senolytic drug.

\section{Background}

Lung cancer is by far the leading cause of cancer death worldwide, with an estimated 1.8 million deaths (about $18 \%$ ) in 2020 [1]. More than $85 \%$ of those cases are currently classified as non-small-cell lung cancer (NSCLC), for which the predicted 5-year survival rate is $15.9 \%$ - a figure that has only marginally improved during the past few decades[2]. In recent years, with the emergence of molecular targeted drugs and immunotherapy drugs, the treatment of NSCLC has made great progress. The main therapy for NSCLC is targeted therapy, approximately $1 / 4$ of NSCLC patients can receive treatment with various targeted drugs, such as gefitinib, erlotinib, afatinib, and crizotinib[3, 4]. However, these drugs only function in a small proportion of patients who harbored specific genetic aberrations and mutation. In addition, patients tend to develop resistance to these drugs[4,5]. Thus, exploring the new therapeutic targets and drugs for NSCLC patients is critically needed.

Skp2 is an F box-type protein and is one of the components of the Skp2-Skp1-Cullin-1-F-box (SCF) ubiquitin ligase quaternary complex [18]. The Skp2 gene expresses the essential protein components of the SCF-Skp2 complex, which can induce protein ubiquitination and subsequent proteasome-dependent degradation [19]. By analyzing the protein substrates of Skp2 (p27, p21, FOX01, p16, Cyclin D/E, BRCA2), it can be inferred that Skp2 functions as an oncogene and participates in many processes of tumor, such 
as cell cycle, metastasis, apoptosis and senescence [6-9]. Skp2 are widely expressed in various solid tumors or hematological malignancies including head and neck cancer, liver cancer, lung cancer, ovary cancer, leukemia, lymphoma and melanoma, correlated with disease progression and poor survival[1015]. In NSCLC, the amplification of DNA at 5p13, the region where Skp2, CDH6 and PC4 are located, is frequently observed using a comparative genomic hybridization method. The aberration of DNA copy number accounts for the elevated expression of Skp2 in 44\% NSCLC samples [16]. For NSCLC patients with KRas mutation, enhanced Skp2 expression is serves as an independent diagnosis marker for poor survival [17]. Therefore, due to the comprehensive oncogenic function of Skp2, Skp2 inhibitor is likely to benefit for cancer therapy.

In order to find novel and effective Skp2 inhibitors, computer virtual screening and biomolecular interaction analysis (Biacore) technology were used to obtain a new type of Skp2 small molecule inhibitors with methylene hydrazide skeleton. Subsequently, we studied the structure-activity relationship and optimized the structure of these compounds. Among them, compound AAA-237 exhibited a significant interaction with Skp2 protein and showed a good anti-tumor activity in cancer cells, especially in NSCLC.

In this study, we further investigated the mechanisms of AAA-237 in NSCLC both in vivo and in vitro. It was found that AAA-237 inhibited the proliferation,DNA synthesis $\square$ colony formation of human NSCLC cells A549 and H1299. In addition, AAA-237 reduced migration and invasion of A549 and H1299 via Skp2/Twist axis. AAA-237 arrested cell cycle at G0/G1 phase through regulating the Skp2-Cip/Kip and $\mathrm{PI} 3 \mathrm{~K} / \mathrm{Akt}-\mathrm{FOXO1}$ signaling pathways. Furthermore, we found that treatment of high concentration AAA237 could induce apoptosis of NSCLC cells via the mitochondrial pathway, however, treatment of low concentration AAA-237 for long-term could induce senescence of NSCLC cells. The same mechanism was also found in A549 xenograft nude mice model. AAA-237 inhibited the growth of tumor by inducing apoptosis and senescence in dose-dependent manner. Taken together, all these results suggested that AAA-237 might be a promising therapeutic agent of NSCLC.

\section{Materials And Methods \\ 2.1 Cell culture}

Human NSCLC cell lines A549 and H1299 were purchased from the GuangZhou Jennio Biotech Co., Ltd. (Guangdong, China). Cells were cultured in humidified incubator with an atmosphere containing $5 \% \mathrm{CO}_{2}$ at $37^{\circ} \mathrm{C}$ and maintained in Dulbecco's Modified Eagle's Medium (DMEM) supplemented with $10 \%$ FBS and antibiotics $(0.1 \mu \mathrm{g} / \mathrm{ml}$ of penicillin and $0.1 \mu \mathrm{g} / \mathrm{ml}$ of streptomycin).

\subsection{Cell proliferation assay}

CCK-8 Kit (Beyotime, China) was used to check cell proliferation according to the manufacturer's instructions. Briefly, cells were seeded in 96-well plates at the density of $3 \times 10^{3}$ per well, cultured for 24 hours and then treated with AAA- 237 for $24 \mathrm{~h}, 48 \mathrm{~h}$ and $72 \mathrm{~h}$. CCK-8 was added and further incubated for 
1h. The absorbance value was measured with a SpectraMax M5 plate reader (Molecular Devices) at 450 $\mathrm{nm}$. IC $\mathrm{I}_{50}$ values were calculated using the GraphPad Prism 7 software (GraphPad Software Inc., San Diego, California, USA).

\subsection{Edu-DNA synthesis assay}

EdU Apollo 567 In Vitro Imaging Kit (RiboBio, Guangzhou, China) was used to assess DNA synthesis according to the manufacturer's instructions. Briefly, cells were seeded in 96-well plates at a density of $5 \times$ $10^{3}$ per well, cultured for 24 hours and then treated with AAA-237 at the concentration of $0.3 \mu \mathrm{M}, 1 \mu \mathrm{M}$ and $3 \mu \mathrm{M}$ for $48 \mathrm{~h}$. EdU was added at a concentration of $50 \mu \mathrm{M}$ and incubated for $2 \mathrm{~h}$ in $37^{\circ} \mathrm{C}$. After that, the cells were fixed in $4 \%$ paraformaldehyde for 30 minutes, permeated with $0.5 \%$ Trixon-X 100 for 10 minutes, and then stained with $10 \mu \mathrm{M}$ Apollo 567 for 30 minutes. Cells were then counterstained with Hoechst 33342 for 30 minutes and photographed by fluorescence microscopy (Nikon Eclipse Ti-U).

\subsection{Soft agar colony formation assay}

Colony formation assay was used to determine tumorigenicity of A549 and H1299 cells. Briefly, cells were seeded in 6-well plates. $2 \mathrm{ml} 0.7 \%$ agar in complete medium was added as the bottom layer and $1 \mathrm{ml}$ of $0.35 \%$ agar in complete medium containing 3,000 cells with $0.3 \mu \mathrm{M}, 1 \mu \mathrm{M}$ and $3 \mu \mathrm{M}$ of AAA-237 was added as the top layer. After cultured at $37^{\circ} \mathrm{C}$ with $5 \% \mathrm{CO}_{2}$ for 2 weeks, the colonies were stained with MTT (3(4,5-dimethyl-2-thiazolyl)-2,5-diphenyl-2-H-tetrazolium bromide) solution (200 $\mu \mathrm{L} /$ well) and photographed.

\subsection{Hoechst 33342 staining assay}

Hoechst 33342 staining assay was carried out to check morphological characteristics of A549 and H1299 cells. Briefly, cells were seeded in 96-well plates at a density of $5 \times 10^{3}$ per well, cultured for 24 hours and then treated with AAA-237 at the concentration of $0.3 \mu \mathrm{M}, 1 \mu \mathrm{M}$ and $3 \mu \mathrm{M}$ for $48 \mathrm{~h}$. Cells were washed with PBS, fixed with $4 \%$ paraformaldehyde, permeabilized with $0.1 \%$ Triton X-100, and stained with $1 \mu \mathrm{g} / \mathrm{ml}$ of Hochest 33342 solution for $30 \mathrm{~min}$ and photographed by fluorescence microscopy (Nikon Eclipse Ti-U).

\subsection{Flow cytometric analysis of apoptosis with Annexin V- FITC/PI staining}

TransDetect Annexin V-FITC/PI cell apoptosis detection kit (TransGen, Beijing, China) was used to check apoptosis rate by Flow cytometer according to the manufacturer's instructions. Briefly, cells were seeded in $6 \mathrm{~cm}$ dishes, cultured for 24 hours and then treated with AAA-237 at the concentration of $0.3 \mu \mathrm{M}, 1 \mu \mathrm{M}$ and $3 \mu \mathrm{M}$ for $48 \mathrm{~h}$. After harvested, cells were incubated with Annexin V-FITC and PI for $15 \mathrm{~min}$ at room temperature. Flow cytometry (BD Accuri C6, San Diego, CA, USA) was performed to analyze the cell apoptosis. Data were analyzed by using Flow Jo Software ((FlowJo X 10).

\subsection{Analysis of mitochondrial membrane potential (MMP, $\Delta \psi \mathrm{m})$}


5,5',6,6'-tetrachloro-1,1',3,3'-tetraethylbenzimidazolocarbocyanine iodide (JC-1) staining was used to check the changes of mitochondrial membrane potential. Cells were seeded in $6 \mathrm{~cm}$ dishes, cultured for $24 \mathrm{~h}$ and treated with AAA-237 at the concentration of $0.3 \mu \mathrm{M}, 1 \mu \mathrm{M}$ and $3 \mu \mathrm{M}$ for $48 \mathrm{~h}$. Then cells were incubated with JC-1 at a final concentration of $10 \mu \mathrm{g} / \mathrm{ml}$ for 20 mins and washed with PBS for 3 times and photographed by fluorescence microscopy (Nikon Eclipse Ti-U).

\subsection{Analysis of caspase 3/7 activity}

Caspase 3/7 live cell fluorescence real-time detection kit (KeyGen BioTECH, Nanjing, China) was used to detect caspase $3 / 7$ activity. Briefly, cells were seeded in $6 \mathrm{~cm}$ dishes, cultured for $24 \mathrm{~h}$ and then treated with AAA-237 at the concentration of $0.3 \mu \mathrm{M}, 1 \mu \mathrm{M}$ and $3 \mu \mathrm{M}$ for $48 \mathrm{~h}$. After harvested, cells were incubated with caspase $3 / 7$ test solution for 30 mins. Flow cytometry (BD Accuri C6, San Diego, CA, USA) was performed to analyze the activity of Caspase $3 / 7$. Data were analyzed by using Flow Jo Software ((FlowJo X 10).

\subsection{RNA sequencing}

A549 cells were seeded in $6 \mathrm{~cm}$ dishes, cultured for 24h and then treated with AAA-237 at the concentration of $1 \mu \mathrm{M}$ for $48 \mathrm{~h}$. Then, cells were dissolved in TRIzol Reagent (Life Technologies, USA) and sent to Novogene Corporation (Beijing, China) for next generation sequencing. RNA integrity was assessed using the RNA Nano 6000 Assay Kit of the Bioanalyzer 2100 system (Agilent Technologies, CA, USA). A total amount of $1 \mu \mathrm{g}$ RNA per sample was used as input material for library preparation and library quality was assessed on the Agilent Bioanalyzer 2100 system. The clustering of the index-coded samples was performed on a cBot Cluster Generation System using TruSeq PE Cluster Kit v3-cBot-HS (Illumia) according to the manufacturer's instructions. After cluster generation, the library preparations were sequenced on an Illumina Novaseq platform and 150 bp paired-end reads were generated.

\subsection{Differentially expressed genes Identification}

FeatureCounts v1.5.0-p3 was used to count the reads numbers mapped to each gene. And then FPKM of each gene was calculated based on the length of the gene and reads count mapped to this gene.

Differential expression analysis of two groups was performed using the DESeq2 R package (1.20.0). The resulting $P$-values were adjusted using the Benjamini and Hochberg's approach for controlling the false discovery rate. Corrected P-value of 0.05 and absolute fold change of 2 were set as the threshold for significantly differential expression.

\subsection{Gene ontology and pathway enrichment analyses}

DAVID website (https://david.ncifcrf.gov/home.jsp) was used to perform gene ontology and pathway enrichment analyses $[18,19]$. The gene ontology terms were recruited from the GOA dataset. The biological pathways were enriched from the KEGG datasets. Cytoscape 3.6.0 was used to visualize coexpression modules[20]. 


\subsection{TIMER2.0 database analysis}

TIMER2.0 (http://timer.cistrome.org/) is a comprehensive database for systematic analysis of immune infiltration in 32 different cancer types from TCGA[21]. TIMER2.0 was used to analyze the correlation between SASP-associated cytokines expression and immune infiltration (CD8 ${ }^{+} \mathrm{T}$ cells, monocytes and macrophages) in lung cancer.

\subsection{RNA extraction, cDNA synthesis and qRT-PCR}

Cells were seeded in $6 \mathrm{~cm}$ dishes, cultured for $24 \mathrm{~h}$ and then treated with AAA-237 at the concentration of $0.3 \mu \mathrm{M}, 1 \mu \mathrm{M}$ and $3 \mu \mathrm{M}$ for $48 \mathrm{~h}$, then harvested in Trizol. After mixing with $1 / 5$ volume of chloroform, the mixture was centrifuged at $12000 \mathrm{rpm}$ for 15 minutes and supernatants were transferred and an equal volume of isopropanol was added to each supernatant and gently mixed. After incubation at room temperature for 30 minutes, the mixture was centrifuged at $12000 \mathrm{rpm}$ for 15 minutes. The pellets were washed once with $75 \%$ ethanol and dissolved in RNase-free water at an appropriate volume. After RNA quantification, cDNA was synthesized using MonScript ${ }^{\mathrm{TM}}$ 5X RTIII All-in-one Mix (Monad Biotech, Wuhan, China) according to the manufacturer's instructions. Quantitative real-time RT-PCR (qRT-PCR) was performed using 2X Taq Pro Universal SYBR qPCR Master Mix (Vazyme Biotech, Nanjing, China). The primers used are listed in Table S1. GAPDH was served as internal control

\subsection{Immunofluroescence}

Cells were seeded into 96 -well plates, cultured for $24 \mathrm{~h}$ and then treated with AAA-237 at the concentration of $0.3 \mu \mathrm{M}, 1 \mu \mathrm{M}$ and $3 \mu \mathrm{M}$ for $48 \mathrm{~h}$. Cells were fixed with $4 \%$ paraformaldehyde for 15 minutes at room temperature, washed with PBS and blocked with 5\% BSA (w/v) and 0.3\% Triton X-100 (v/v) for 1 hour at room temperature. Cells were then incubated with the indicated primary antibody (Table S2) diluted with PBS containing $1 \% \mathrm{BSA}(\mathrm{w} / \mathrm{v})$ overnight at $4^{\circ} \mathrm{C}$. Cells were washed three times with PBS and incubated with the corresponding fluorescent secondary antibody for $2 \mathrm{~h}$ at room temperature. After three washes with PBS, cells were stained with $10 \mu \mathrm{g} / \mathrm{ml}$ Hoechst 33342 for 30 minutes, washed with PBS and photographed by fluorescence microscopy (Nikon Eclipse Ti-U).

\subsection{Western blotting}

Cells were seeded in $6 \mathrm{~cm}$ dishes, cultured for $24 \mathrm{~h}$ and then treated with AAA-237 at the concentration of $0.3 \mu \mathrm{M}, 1 \mu \mathrm{M}$ and $3 \mu \mathrm{M}$ for $24 \mathrm{~h}, 48 \mathrm{~h}$ and $72 \mathrm{~h}$. Cells were harvested and lysed with RIPA (Applygen, Beijing, China) lysis buffer at $4^{\circ} \mathrm{C}$ for 30 minutes for the total protein. BCA method (Beyotime, Guangzhou, China) was used to measure the concentration of protein. Equivalent amounts of protein were loaded and separated by $10 \%$ SDS-PAGE and then transferred to a PVDF membrane (Millipore, Billerica, MA). The membranes were blocked in $5 \%$ fat-free milk for $2 \mathrm{~h}$ at room temperature, then incubated with primary 
antibodies (Table S2) overnight at $4^{\circ} \mathrm{C}$. Then, the membrane was incubated with goat anti-mouse or antirabbit HRP-conjugated secondary antibody (CWBIO, Beijing, China). Finally, immunoreactive bands were visualized using ECL Western Blot Kit (CWBIO, Beijing, China).

\subsection{SA- $\beta$-Gal Assay}

A senescence detection kit (BioVision Inc., Milpitas, CA, USA) was used to detect senescence-associated $\beta$-galactosidase (SA- $\beta$-Gal) activity. Briefly, cells were seeded in $12 \mathrm{~cm}$ dishes, cultured for $24 \mathrm{~h}$ and then treated with AAA-237 at the concentration of $0.3 \mu \mathrm{M}$ for 1 day, 3 days and 7 days. Cells were washed twice with PBS, exposed to fixation solution for $15 \mathrm{~min}$, and then incubated overnight in freshly prepared staining solution and then examined microscopically ((Nikon Eclipse Ti-U).

\subsection{In vivo anti-tumor activity}

Six week-old female BALB/c-nu nude mice (Beijing Vital River Laboratory Animal Technology Co., Ltd., Beijing, China) weighing 15-18 g were housed in barrier facilities with a $12 \mathrm{~h}$ light/dark cycle. All experimental procedures were carried out in accordance with institutional guidelines for the care and use of laboratory animals at the Institute of Materia Medica, Chinese Academy of Medical Science \& Peking Union Medical College and the National Institutes of Health Guide for Care and Use of Laboratory Animals (publication no. 85-23, revised 1985). Briefly, A549 cells ( $1 \times 10^{7}$ cells per mouse) were subcutaneously implanted into the right flanks of the mice. After 14 days, when the tumor volumes grew to $100 \mathrm{~mm}^{3}$, the mice were divided into 4 groups randomly $(n=6)$, for treatment with vehicle, paclitaxel (20 mg/kg, ip, twice a week, for 2 weeks), AAA-237 low dose (15 mg/kg, ig, once a day, for 2 weeks) or AAA-237 high dose ( $45 \mathrm{mg} / \mathrm{kg}$, ig, once a day, for 2 weeks). The tumor volume was measured using a vernier caliper and calculated according to the modified ellipsoid formula: Tumor volume $\left(\mathrm{mm}^{3}\right)=$ (length) $\times(\text { width })^{2} \times 0.5$. At the end of the experiment, all the mice were sacrificed and the tumor tissues and other organs were harvested, weighted and photographed.

\subsection{Statistical analysis}

Data are presented as mean \pm stand deviation (SD), representative of at least three independent experiments. One-way ANOVA was used to calculate differences between the various study groups. The $p$ Value $<0.05$ was considered statistically significant.

\section{Results}

\subsection{AAA-237 inhibited proliferation of NSCLC cells}


AAA-237 is a Skp2 inhibitor with methylene hydrazide skeleton, which is firstly synthesized by our group (Fig. 1A). Results from Western blotting showed that AAA-237 could significantly inhibit the expression of Skp2 in NSCLC cells (Fig. 1B). To determine the inhibitory effect of AAA-237 on the viability and proliferation of NSCLC cells, CCK8 assay was performed and changes of cell morphology were also recorded. The results showed that AAA-237 inhibited the proliferation of A549 and H1299 cells in dosedependent and time-dependent manner (Fig. 1C). The IC $\mathrm{I}_{50}$ of AAA-237 for the A549 cells was $3 \mu \mathrm{M}$ at 24 $\mathrm{h}, 2.5 \mu \mathrm{M}$ at $48 \mathrm{~h}$ and $0.7 \mu \mathrm{M}$ at $72 \mathrm{~h}$. The $\mathrm{IC}_{50}$ of AAA-237 for the $\mathrm{H} 1299$ cells was $3.9 \mu \mathrm{M}$ at $24 \mathrm{~h}, 1.8 \mu \mathrm{M}$ at $48 \mathrm{~h}$ and $1.1 \mu \mathrm{M}$ at $72 \mathrm{~h}$ (Fig. S1A). The morphology of the A549 and $\mathrm{H} 1299$ cells were significantly changed after treatment of AAA-237 in a dose-dependent manner (Fig. S1B). To check if AAA-237 inhibit DNA synthesis, EdU staining was performed. As shown in Fig. 1D, the DNA synthesis in A549 and H1299 cells were significantly inhibited after treatment of AAA-237 in a dose-dependent manner. To evaluate the effect of AAA-237 on the formation of colonies in NSCLC cells, soft agar colony assays were performed. Results showed that AAA-237 inhibited colony formation of A549 and H1299 cells in the soft agar in a dose-dependent manner (Fig. 1E). Taken together, our results showed that AAA-237 could inhibit the growth of NSCLC cells in vitro.

\subsection{AAA-237 inhibited migration and invasion of NSCLC cells by Skp2/Twist axis}

To determine if AAA-237 could affect migration and invasion of NSCLC cells, Transwell assays were performed. Results showed that AAA-237 significantly inhibited the migration and invasion of A549 and H1299 cells in a dose-dependent manner (Fig. 2A-B). E-cadherin, N-cadherin, Vimentin and matrix metallopeptidases (MMPs) were well-known regulators in EMT, which was closely associated with migration and invasion by degrading and remodeling extracellular matrix. Results from Western blotting showed that the expression of MMP7, N-cadherin and Vimentin was reduced while the expression of Ecadherin was increased after AAA-237 treatment in A549 and H1299 cells (Fig. 2C). Twist was a major transcription factor which mediated EMT. Skp2 was reported to facilitate EMT process through stabilizing Twist, which also raised the possibility that Skp2/Twist axis acted as another arm to drive cell migration, invasion and cancer metastasis[22]. Expression of Twist was checked by Western blotting and it was shown in Fig. 2C that AAA-237 inhibited the expression of Twist in A549 and H1299 cells. Taken together, these results suggest that AAA-237 inhibited the process of EMT by regulating Twist expression to block the metastatic biological functions of NSCLC cells (Fig. 2D).

\subsection{Differential gene expression and enrichment analysis in A549 cells treated with AAA-237}

To further explore potential mechanisms of AAA-237 on NSCLC, differentially expressed genes of A549 cells treated with $1 \mu \mathrm{M}$ AAA-237 for $48 \mathrm{~h}$ and control cells were identified by RNA-seq assay. Compared with gene expression in the control cells, 757 up-regulated genes and 1242 down-regulated genes were found in the cells treated with AAA-237 ( $|\log 2 \mathrm{FC}| \geq 1$ and $P$-value $\leq 0.05$, Fig. 3A-B). 
The results from the Gene Ontology enrichment analyses of these differentially expressed genes showed that the genes enriched in biological processes (BP) category involved the regulation of ERK1 and ERK2 cascade, regulation of signaling receptor activity and response to lipopolysaccharide (Fig. $3 \mathrm{C}$ ). In the cell components (CC) category, the differentially expressed genes were involved in proteinaceous extracellular matrix, MCM complex and chromocenter (Fig. 3C). In the molecular function (MF) category, the differentially expressed genes were involved in hijacked molecular function, virus receptor activity and carboxylic acid binding (Fig. 3C). The results from the KEGG pathway enrichment analysis showed that the genes were enriched in pathways that involved in cancers, cellular senescence, DNA replication, cell cycle, FOXO signaling pathway (Fig. 3D-E).

\subsection{AAA-237 arrested cell cycle at G0/G1 checkpoint by regulating the Skp2-Cip/Kip and PI3K/Akt-FOXO1 signaling pathway}

Cell cycle assay was performed and results showed that arrest of cell cycle occurred at the G0/G1 phase in A549 and H1299 cells in a dose- and time-dependent manner (Fig. 4A). To investigate the underlying mechanism of AAA-237, the expression level of the proteins associated with G0/G1 cell cycle checkpoints in A549 and H1299 cells treated with AAA-237 was checked by Western blotting. It was shown in Fig. 4B that the expression level of CDK2, cyclin E1, CDK4, and cyclin D was reduced in A549 and H1299 cells. The roles of Cip/Kips family of cyclin-dependent kinase inhibitors (CKI), such as p21 Cip1 and p27 Kip1, in negatively regulating the cell cycle and in genomic stability, apoptosis, senescence and DNA repair have been well characterized[23, 24]. Skp2 played critical roles in mediating the ubiquitin-dependent degradation of those CKIs, such as p21 Cip1 and p27 Kip1. Our results showed that the expression of CKls p21 Cip1 and p27 Kip1 were increased after treatment of Skp2 inhibitor AAA-237 in A549 and H1299 cells in dose-dependent and time-dependent manner, which indicated that AAA-237 could regulate Skp2Cip/Kip pathway, therefore suppress CDK 2/cyclin E and CDK 4/cyclin D complexes in NSCLC cells (Fig. 4B).

Previous studies showed that PI3K/Akt signaling pathway promoted the phosphorylation of FOXO1 and FOX01 was transferred from the nucleus to the cytoplasm, which resulted in the loss of transcriptional activity, thereby inhibited the expression of downstream genes regulated by FOX01[25-27]. Our results showed that AAA-237 reduced the phosphorylation levels of PI3K and Akt in A549 and H1299 cells. Moreover, Western blotting and immunofluorescence analysis indicated that nuclear FOXO1, which represents the transcriptional activity of this molecule, exhibited an upregulated level, therefore promoted the expression of downstream genes including p15 INK4B, p21 Cip1 and p27 Kip1 in A549 and H1299 cells (Fig. 4C, Fig. S2). Overall, these results suggested that AAA-237 could inhibit the PI3K/Akt signaling pathway and enhance the activity of the transcription factor FOXO1 in A549 and $\mathrm{H} 1299$ cells, thereby regulating the expression of p15 INK4B, p21 Cip1 and p27 Kip1 at transcription level, and promoting arresting of cell cycle at the G0/G1 phase in NSCLC cells. 


\subsection{AAA-237 induced apoptosis of the NSCLC cells in a mitochondria dependent manner}

To explore whether AAA-237 induced apoptosis of NSCLC cells, A549 and H1299 were stained with Hoechst 33342 dye. It was shown in Fig. S1C that the nuclei of the A549 and H1299 cells were concentrated and aggregated after treatment with AAA-237, and vacuoles appeared, which means these cells were at the early stage of apoptosis. Furthermore, Annexin V-EGFP/PI double staining was used and apoptosis was analyzed by Flow cytometry. It was found that AAA-237 induced apoptosis of the A549 and H1299 cells in dose- and time-dependent manner (Fig. 5A). To further explore the mechanism of apoptosis induced by AAA-237, the changes in mitochondrial membrane potential (MMP, $\Delta \psi m$ ) and the activity of caspase-3/7 was measured. JC- 1 staining was used to determine MMP after treatment of AAA237 for $48 \mathrm{~h}$ in $\mathrm{A} 549$ and $\mathrm{H} 1299$ cells. Result showed that the ratio of JC-1 monomer/JC-1 polymer was increased in A549 and H1299 cells as the concentration of AAA-237 was increased (Fig. 5B). The activity of caspase-3/7 was also determined by Flow cytometry. As shown in Fig. 5C, the caspase-3/7 activity was increased after treatment of AAA-237 in A549 and H1299 cells. The expression of apoptosis-related proteins was also checked by Western blotting. Results showed that the expression level of cleaved PARP, cleaved caspase 3, cleaved caspase 9 and Bax was increased and the expression level of PARP and Bcl-2 was decreased after treatment of AAA-237 in A549 and H1299 cells (Fig. 5D), which suggested that AAA237 may induced apoptosis in the mitochondrial pathway by up-regulating pro-apoptotic protein Bax and down-regulating anti-apoptotic protein Bcl-2. Together, these results suggest that AAA-237 induced apoptosis in a mitochondria dependent manner in NSCLC cells.

\subsection{AAA-237 induced cellular senescence of the NSCLC cells}

To our surprise, after treatment of AAA-237, some NSCLC cells exhibited an enlarged nuclei morphology (Fig. S1C). Such changes may occur in senescent cells [49]. To investigate whether AAA-237 induced cellular senescence, we detected the activity of SA- $\beta$-Gal, a well-known marker for senescent cell. Result showed that treatment of low concentration AAA-237 in A549 and H1299 cells for long-term ( 3 days and 7 days) could increase the number of SA- $\beta$-Gal-positive cells compared with the control group (Fig. 6A). Studies have revealed that Skp2 is also involved in DDR mechanisms[28, 29].In our study, we found that AAA-237 inhibited the expression of Skp2, which lead to the DNA damage. Immunofluorescence results showed the phosphorylation of $\mathrm{H} 2 \mathrm{AX}(\mathrm{YH} 2 \mathrm{AX})$, which is an early sign of DNA damage as well as a senescence marker [50,51], was increased in A549 and H1299 cells after treatment of AAA-237 for longterm (Fig. 6B). Result from Western blotting showed that the expression of $\mathrm{yH} 2 \mathrm{AX}$ and $\mathrm{p} 16$, another marker of senescence, was increased in A549 and H1299 cells after treatment of AAA-237 (Fig. 6C). Senescent cells may exhibit a senescence-associated secretory phenotype (SASP), a process whereby high levels of SASP factors are secreted [52]. PCR result showed that after treatment of AAA-237 for longterm, the level of SASP-associated cytokines (IL-1A, IL-6, CXCL1, CXCL8, MMP1 and MMP10) was significantly increased in A549 and H1299 cells (Fig. 6D-E). Taken together, these results suggest that 
AAA-237 induced senescence of A549 and H1299 cells through DNA damage and exhibited a SASP phenotype.

Senescent cells in tumors have immune activation properties by secreting SASP-associated cytokines to promote the recruitment and activation of immune cells in tumor microenvironment. Therefore, we assessed the correlation of SASP-associated cytokines expression with immune infiltration levels in lung cancer by TIMER. The heatmap result also indicated that SASP-associated cytokines IL-1A, IL-6, CXCL1, MMP1 and MMP10 was associated with immune infiltration (Fig S3A). The expression of IL-1A, IL-6, MMP1 and MMP10 were positively associated with the infiltration levels of CD $8^{+} T$ cells, monocytes and macrophages (Figure S3B-E).

However, the accumulation of senescent cells in the tumor microenvironment will largely lead to tumor recurrence and metastasis. Quercetin is a senolytic drug that can selectively eliminate senescent cells [54]. Therefore, the effects of quercetin on the viability of AAA-237-induced senescent A549 and H1299 cells were examined. As showed in Fig. 7A, quercetin significantly decreased the viability of AAA-237treated $\mathrm{A} 549$ and $\mathrm{H} 1299$ cells especially in high concentration $(60 \mu \mathrm{M})$. Thus, the senolytic drug, quercetin, was effective in NSCLC cells in which AAA-237 induced senescent changes.

In short, our results suggested that treatment of low concentration AAA-237 could induce senescence through DNA damage. Senescent cells secreted SASP-associated cytokines IL-1A, IL-6, CXCL1, CXCL8, MMP1 and MMP10, which recruiting and activating CD8 + T cells, monocytes and macrophages. Senolytic drug quercetin could selectively eliminate senescent A549 and H1299 cells (Fig. 7B).

\subsection{AAA-237 suppressed tumorigenicity in vivo}

To explore whether AAA-237 has anti-NSCLC activity in vivo, a xenograft model was established by inoculating nude mice with A549 cells and then treated with 15 or $45 \mathrm{mg} / \mathrm{kg}$ of AAA-237 every day for 14 days or $20 \mathrm{mg} / \mathrm{kg}$ of paclitaxel per mouse twice a week for 14 days. As shown in Fig. 8A-C, the intraperitoneal administration of AAA-237 significantly reduced volume and weight of tumor. The tumor growth inhibition (TGI) of paclitaxel group, AAA-237 low and AAA-237 high is $48 \%, 55 \%$ and $64 \%$ (Table 1). These results indicated that AAA-237 could inhibit growth of the tumor in a dose-dependent manner, and the anti-tumor effect of AAA-237 is better than the positive drug paclitaxel. During the treatment of AAA-237, no obvious weight loss or abnormal behavior was observed in the nude mice (Fig. 8D). Furthermore, no mortality or significant changes in the colors and textures of vital organs, including the heart, liver, spleen, lung and kidney and no significant differences in the relative organ weights were observed in the AAA-237-treatment groups compared to control group (Fig. 8E). No obvious systemic toxicity was observed in physiological blood tests (Fig. S4, Table S3). 
Table 1

Antitumor activity of AAA-E37 in lung cancer A549 xenograft mice model.

\begin{tabular}{|lllll|}
\hline Group & Dose $/ \mathbf{m g} \cdot \mathbf{k g}^{-1}$ & Number (end / start) & Tumor weight / g & TGI (\%) \\
\hline Control & - & $6 / 6$ & $0.39 \pm 0.09$ & - \\
\hline Tax & 20 & $6 / 6$ & $0.20 \pm 0.06 * *$ & 48 \\
\hline AAA-E37-low & 15 & $6 / 6$ & $0.18 \pm 0.05 * * *$ & 55 \\
\hline AAA-E37-high & 45 & $6 / 6$ & $0.14 \pm 0.03^{\star * * *}$ & 64 \\
\hline $\begin{array}{l}\mathrm{n}=6 \text {, mean } \pm \text { SD. } \\
\text { inhibition; }\end{array}$ & & & & \\
\hline
\end{tabular}

In order to explore the mechanism of AAA-237 in vivo, we carried out Ki67 immunohistochemistry and TUNEL (TdT mediated dUTP Nick End Labeling) fluorescence staining in tumor tissues. Ki67 is a proliferation-related antigen which is closely related to mitosis and is indispensable in cell

proliferation[30]. Results from immunohistochemistry showed that the expression of Ki67 in tumor tissue was reduced after treatment of AAA-237, which indicated that AAA-237 could inhibited the proliferation of cancer cell in vivo (Fig. 9A). TUNEL staining can be used to detect the nuclear DNA breakage of tissue in the late stage of apoptosis. We found that treatment of AAA-237 promoted nuclear DNA breakage, therefore induced cell apoptosis in tumor tissue (Fig. 9B). Results from Western blotting also showed that AAA-237 inhibited the expression of Skp2, up-regulating the expression of p27 Kip1 in tumor tissue, therefore inhibited growth of tumor. AAA-237 also induced apoptosis of tumor cells by cleaving PARP, caspase 9 and caspase 3 , increasing expression of Bax and decreasing expression of $\mathrm{Bcl}-2$. The expression of senescence-related proteins $\mathrm{\gamma H} 2 \mathrm{~A} . \mathrm{X}$ and p16 is also significantly up-regulated, indicating that AAA-237 can also induce senescence of tumor cells (Fig. 9C). Taken together, our results suggested that AAA-237 inhibited growth of tumor by regulating Skp2-Kip pathway and inducing apoptosis and senescence of tumor cells in vivo.

\section{Discussion}

Lung cancer is the leading cause of cancer death worldwide, and most of patients with lung cancer are non-small cell lung cancer (NSCLC). NSCLC is still very difficult to treat. Skp2 expression is identified to be a useful diagnosis marker of NSCLC patients[17]. Skp2 participates in many cell processes in tumors, such as cell cycle, proliferation, metastasis, and apoptosis[6-8]. Since most of the Skp2 substrates are tumor suppressor proteins, it can be asserted that Skp2 functions as an oncogene[8, 31, 32]. AAA-237, a small molecule Skp2 inhibitor with methylene hydrazide skeleton, was firstly synthesized by our group. AAA-237 was found to have a significant inhibitory effect on different kinds of cancer cells, especially on NSCLC cells. In this study, we further investigated the effect of AAA-237 on NSCLC in vitro and in vivo and explored the underlying molecular mechanisms in details. Our results showed that AAA-237 inhibited proliferation, decreased DNA synthesis and inhibited colony formation of A549 and H1299 cells. AAA-237 also inhibited the migration and invasion by regulating Skp2/Twist axis. AAA-237 induced arresting of 
the cell cycle at the G0/G1 phase, which is regulated by Skp2-Cip/Kip and PI3K/Akt-FOXO1 signaling pathway. Furthermore, we found that treatment of high concentration AAA-237 could induce apoptosis in a mitochondria dependent manner, however, treatment of low concentration AAA-237 for long-term could induce senescence through DNA damage and exhibited a SASP phenotype. The same mechanism was also found in A549 xenograft nude mice model, AAA-237 dose-dependently inhibited the growth of tumor by inducing apoptosis and senescence. Taken together, these results suggest that Skp2 inhibitor AAA-237 could be used as apoptosis and senescence inducer with potential to be a novel anti-NSCLC agent.

Metastasis is the leading cause of mortality in human NSCLC[33]. Tumor metastasis is a complicated process and EMT plays an important role in this process[34,35]. EMT is a process which cancer cells lose epithelial features, cell shape changes and cells activate genes that help to define mesenchymal phenotype, what leads to an increased cell motility and dissemination of tumor to distant metastatic sites[36]. When EMT occurs, the cells will lose the expression of E-cadherin, acquire the expression of $\mathrm{N}$ cadherin and Vimentin and increase the expression of MMPs[35, 37]. The process of EMT is controlled by EMT-related transcription factor, such as twist $[36,38,39]$. Here, our results showed that the expression of E-cadherin was introduced and expression of MMP7, N-cadherin and Vimentin was reduced in NSCLC cells treated by AAA-237 through inhibiting the expression of transcription factor twist. AAA-237 may inhibit the migration and invasion of NSCLC by regulating Skp2/Twist axis.

The most famous substrates of Skp2 are the cell cycle inhibitors p21 Cip1 and p27 Kip1. Overexpression of Skp2 induced p21 Cip1 and p27 Kip1 ubiquitination and continuous degradation of p21 Cip1 and p27 Kip1, while low levels of Skp2 reduced their degradation. In our study, we found that after treatment of AAA-237, the expression of p21 Cip1 and p27 Kip1 in A549 and H1299 cells was increased. In addition, we enriched and analyzed the differential genes obtained from RNA-seq and found that the FOXO1 signaling pathway was involved in the regulation of cell cycle after treatment of AAA-237. It is known that the prominent role of transcription factor FOXO1 in arresting of cell cycle depended on the expression of its targets, such as p15 INK4B, p21 Cip1 and p27 Kip1 [40, 41], which initiated significant inhibition of cell proliferation. In our study, we further verified the regulatory effect of FOXO1 signaling pathway on arresting of cell cycle after AAA-237 treatment and demonstrated that AAA-237 increased the expression of nucleus FOXO1 by inhibiting the activation of PI3K/Akt signaling pathway, therefore improved its transcriptional activity and increased the expression of p15 INK4B, p21 Cip1 and p27 Kip1 at the transcription level and induced arrest of cell cycle.

One important finding in the study is that treatment of low concentration AAA-237 in cells for long time induced senescence of NSCLC cells. Senescence is an inevitable phenomenon in the survival and growth of organisms [42]. DNA damage response (DDR) signaling is critical for DNA damage-induced senescence[43]. The increased activity of $\mathrm{YH} 2 \mathrm{AX}$, the phosphorylation form of histone $\mathrm{H} 2 \mathrm{~A}$ member $\mathrm{X}$ at serine 139, in response to DDR critically regulates cellular senescence and has been considered as a biomarker of cellular senescence. Studies have revealed that Skp2 is also involved in DDR mechanisms $[28,29]$.In our study, we found that AAA-237 inhibited the expression of Skp2, which lead to 
the DNA damage. Accumulation of nuclear DNA damage stimulates the activity of $\mathrm{yH} 2 \mathrm{AX}$, and then triggers the initiation of cellular senescence[44].

Senescent cells are usually characterized by arrest of cell cycle and the production of SASP phenotype [45]. The tumor suppressor protein p16 Ink4a is often transcriptionally activated in cells undergoing senescence and is one of the main regulators of this program[46]. In senescent cells, arrest of cell cycle correlates with an augmented level of p16 INK4a [47]. In our study, we found that the expression level of p16 Ink4a was increased after treatment of AAA-237, which indicated that DNA damage caused by AAA237, may activate p16 INK4a. The p16 INK4a mediated senescence through activating p21 CIP1, which induced the arrest of cell cycle at G0/G1 phase[48].

In contrast to apoptotic cells, senescent cells are metabolically active and may affect other cells via secretion of multiple inflammatory proteins, described as the SASP[49]. SASP molecules including CCL2, CCL5, IL7, and IL15 can recruit and activate innate and acquired immune cells, such as NK cells, monocytes, macrophages and T cells. In addition, these cells can transmit signals to immune cells, such as neutrophils, $B$ cells, and dendritic cells after being activated, thereby establishing an immune activation microenvironment[50]. Therefore, the induction of senescence can not only induce arrest of cell cycle, but also mobilize immune surveillance in tumor. Induction of senescence of cancer cell become a promising method in current cancer treatment. In our study, we demonstrated that AAA-237 induced senescence of NSCLC cells. AAA-237 increased the activity of SA- $\beta-G$ al and the expression of senescence markers $\mathrm{YH} 2 \mathrm{~A} . \mathrm{X}$ and $\mathrm{p} 16$. The secretion of SASP-associated cytokines including IL-1A, IL-6, CXCL1, CXCL8, MMP1 and MMP10 was increased in cells treated by AAA-237 induced, which further promoted the infiltration levels of $\mathrm{CD} 8^{+} \mathrm{T}$ cells, monocytes and macrophages in lung cancer.

However, the accumulation of senescent cells in the tumor microenvironment would largely lead to tumor metastasis. The pro-inflammatory SASP produced by senescent cells greatly promotes the proliferation and migration of tumor cells. Therefore, long-existing senescent cells have brought challenges to the application of tumor senescence therapy. Senolytic drug is a kind of drug that selectively kills senescent cells[51, 52] [53]. It can effectively eliminate senescent cells in the human body to reduce its harmful effects and maintain normal functions of the body. Thus, combining senescence-inducing therapy with senolytic drugs could be beneficial to short and long-term outcomes in cancer patients[42, 54]. Here, we demonstrated that AAA-237 induced senescence in some cases. Senolytic drug quercetin could selectively eliminate senescent A549 and H1299 cells. Thus, combining AAA-237-induced senescence therapy with senolytic drug quercetin could be beneficial to NSCLC patients.

\section{Conclusion}

In summary, we demonstrated that Skp2 inhibitor AAA-237 exhibited significant growth inhibitory effect on NSCLC cells in vitro and in vivo, which was associated with arresting of cell cycle at G0/G1 phase by regulating the Skp2-Cip/Kip and PI3K/Akt-FOXO1 signaling pathways. AAA-237 also exerted anti-tumor 
effects on NSCLC by inducing mitochondria-dependent apoptosis and DNA damage-induced senescence. Thus, we think that the novel Skp2 inhibitor AAA-237 might be a promising therapeutic agent of NSCLC.

\section{Declarations}

\section{Ethics approval and consent to participate}

This study was approved by the ethics committee for laboratory animal care and use of Institute of Materia Medica, CAMS \& PUMC.

\section{Consent for publication}

Not applicable.

\section{Availability of data and materials}

The datasets used and/or analysed during the current study are available from the corresponding author on reasonable request.

\section{Competing interests}

The authors declare that they have no competing interest.

\section{Funding}

This research was funded by Beijing Natural Science Foundation (7212157), CAMS Innovation Fund for Medical Sciences (2016-I2M-3-007), National Natural Science Foundation of China (81803584, 81703536), Technology Major Projects for "Major New Drugs Innovation and Development" (2018ZX09711001-005-025, 2018ZX09711001-012), Natural Science Foundation of China (82073311), the Key Research and Development Projects in Chengdu (2020-YF05-00058-SN), the Key Research and Development Projects in Sichuan Province (2020YFS0399).

\section{Authors' contributions}

Jinhua Wang and Jianyou Shi developed the hypothesis, designed the experiments, and revised the manuscript. Jinyi Liu conducted most functional experiments and wrote the main manuscript. Xiangjin Zheng and Wanli performed the Western blotting, Liwen Ren and Sha Li performed the PCR. Yihui Yang and Binbin Ge performed the statistical analyses. Guanhua Du revised the manuscript. All authors read and approved the final manuscript.

\section{Acknowledgements}

Not applicable. 


\section{References}

1. H. Sung, J. Ferlay, R.L. Siegel, M. Laversanne, I. Soerjomataram, A. Jemal, F. Bray, Global cancer statistics 2020: GLOBOCAN estimates of incidence and mortality worldwide for 36 cancers in 185 countries, CA Cancer J Clin (2021).

2. Z. Chen, C.M. Fillmore, P.S. Hammerman, C.F. Kim, K.K. Wong, Non-small-cell lung cancers: a heterogeneous set of diseases, Nature reviews. Cancer 14(8) (2014) 535-46.

3. E.B. Garon, N.A. Rizvi, R. Hui, N. Leighl, A.S. Balmanoukian, J.P. Eder, A. Patnaik, C. Aggarwal, M. Gubens, L. Horn, E. Carcereny, M.J. Ahn, E. Felip, J.S. Lee, M.D. Hellmann, O. Hamid, J.W. Goldman, J.C. Soria, M. Dolled-Filhart, R.Z. Rutledge, J. Zhang, J.K. Lunceford, R. Rangwala, G.M. Lubiniecki, C. Roach, K. Emancipator, L. Gandhi, Pembrolizumab for the treatment of non-small-cell lung cancer, The New England journal of medicine 372(21) (2015) 2018-28.

4. R.S. Herbst, D. Morgensztern, C. Boshoff, The biology and management of non-small cell lung cancer, Nature 553(7689) (2018) 446-454.

5. D.R. Camidge, W. Pao, L.V. Sequist, Acquired resistance to TKIs in solid tumours: learning from lung cancer, Nature reviews. Clinical oncology 11(8) (2014) 473-81.

6. Z. Wang, D. Gao, H. Fukushima, H. Inuzuka, P. Liu, L. Wan, F.H. Sarkar, W. Wei, Skp2: a novel potential therapeutic target for prostate cancer, Biochimica et biophysica acta 1825(1) (2012) 11-7.

7. D.D. Hershko, Oncogenic properties and prognostic implications of the ubiquitin ligase Skp2 in cancer, Cancer 112(7) (2008) 1415-24.

8. K.I. Nakayama, K. Nakayama, Ubiquitin ligases: cell-cycle control and cancer, Nature reviews. Cancer 6(5) (2006) 369-81.

9. Z. Cai, A. Moten, D. Peng, C.C. Hsu, B.S. Pan, R. Manne, H.Y. Li, H.K. Lin, The Skp2 Pathway: A Critical Target for Cancer Therapy, Seminars in cancer biology 67(Pt 2) (2020) 16-33.

10. D.G. Carracedo, A. Astudillo, J.P. Rodrigo, C. Suarez, M.V. Gonzalez, Skp2, p27kip1 and EGFR assessment in head and neck squamous cell carcinoma: prognostic implications, Oncology reports 20(3) (2008) 589-95.

11. E. Shin, S.H. Kim, H.Y. Jeong, J.J. Jang, K. Lee, Nuclear expression of S-phase kinase-associated protein 2 predicts poor prognosis of hepatocellular carcinoma, APMIS : acta pathologica, microbiologica, et immunologica Scandinavica 120(5) (2012) 349-57.

12. K. Shigemasa, L. Gu, T.J. O'Brien, K. Ohama, Skp2 overexpression is a prognostic factor in patients with ovarian adenocarcinoma, Clinical cancer research : an official journal of the American Association for Cancer Research 9(5) (2003) 1756-63.

13. Y.H. Min, J.W. Cheong, M.H. Lee, J.Y. Kim, S.T. Lee, J.S. Hahn, Y.W. Ko, Elevated S-phase kinaseassociated protein 2 protein expression in acute myelogenous leukemia: its association with constitutive phosphorylation of phosphatase and tensin homologue protein and poor prognosis, Clinical cancer research : an official journal of the American Association for Cancer Research 10(15) (2004) 5123-30. 
14. R. Seki, T. Okamura, H. Koga, K. Yakushiji, M. Hashiguchi, K. Yoshimoto, H. Ogata, R. Imamura, Y. Nakashima, M. Kage, T. Ueno, M. Sata, Prognostic significance of the F-box protein Skp2 expression in diffuse large B-cell lymphoma, American journal of hematology 73(4) (2003) 230-5.

15. Q. Li, M. Murphy, J. Ross, C. Sheehan, J.A. Carlson, Skp2 and p27kip1 expression in melanocytic nevi and melanoma: an inverse relationship, Journal of cutaneous pathology 31(10) (2004) 633-42.

16. S. Yokoi, K. Yasui, M. Mori, T. lizasa, T. Fujisawa, J. Inazawa, Amplification and overexpression of SKP2 are associated with metastasis of non-small-cell lung cancers to lymph nodes, The American journal of pathology 165(1) (2004) 175-80.

17. C.Q. Zhu, F.H. Blackhall, M. Pintilie, P. Iyengar, N. Liu, J. Ho, T. Chomiak, D. Lau, T. Winton, F.A. Shepherd, M.S. Tsao, Skp2 gene copy number aberrations are common in non-small cell lung carcinoma, and its overexpression in tumors with ras mutation is a poor prognostic marker, Clinical cancer research : an official journal of the American Association for Cancer Research 10(6) (2004) 1984-91.

18. W. Huang da, B.T. Sherman, R.A. Lempicki, Systematic and integrative analysis of large gene lists using DAVID bioinformatics resources, Nature protocols 4(1) (2009) 44-57.

19. W. Huang da, B.T. Sherman, R.A. Lempicki, Bioinformatics enrichment tools: paths toward the comprehensive functional analysis of large gene lists, Nucleic acids research 37(1) (2009) 1-13.

20. W. Li, J. Liu, W. Fu, X. Zheng, L. Ren, S. Liu, J. Wang, T. Ji, G. Du, 3-O-acetyl-11-keto-beta-boswellic acid exerts anti-tumor effects in glioblastoma by arresting cell cycle at G2/M phase, Journal of experimental \& clinical cancer research : CR 37(1) (2018) 132.

21. T. Li, J. Fu, Z. Zeng, D. Cohen, J. Li, Q. Chen, B. Li, X.S. Liu, TIMER2.0 for analysis of tumor-infiltrating immune cells, Nucleic acids research 48(W1) (2020) W509-w514.

22. D. Ruan, J. He, C.F. Li, H.J. Lee, J. Liu, H.K. Lin, C.H. Chan, Skp2 deficiency restricts the progression and stem cell features of castration-resistant prostate cancer by destabilizing Twist, Oncogene 36(30) (2017) 4299-4310.

23. S. Lim, P. Kaldis, Cdks, cyclins and CKIs: roles beyond cell cycle regulation, Development (Cambridge, England) 140(15) (2013) 3079-93.

24. C. Denicourt, S.F. Dowdy, Cip/Kip proteins: more than just CDKs inhibitors, Genes \& development 18(8) (2004) 851-5.

25. Y.Q. Xing, A. Li, Y. Yang, X.X. Li, L.N. Zhang, H.C. Guo, The regulation of FOXO1 and its role in disease progression, Life Sci 193 (2018) 124-131.

26. S. Duan, W. Huang, X. Liu, X. Liu, N. Chen, Q. Xu, Y. Hu, W. Song, J. Zhou, IMPDH2 promotes colorectal cancer progression through activation of the PI3K/AKT/mTOR and PI3K/AKT/FOXO1 signaling pathways, Journal of experimental \& clinical cancer research : CR 37(1) (2018) 304.

27. Y. Yan, H. Huang, Interplay Among PI3K/AKT, PTEN/FOXO and AR Signaling in Prostate Cancer, Adv Exp Med Biol 1210 (2019) 319-331.

28. W. Yan, Y. Yang, W. Yang, Inhibition of SKP2 Activity Impaired ATM-Mediated DNA Repair and Enhanced Sensitivity of Cisplatin-Resistant Mantle Cell Lymphoma Cells, Cancer biotherapy \& 
radiopharmaceuticals 34(7) (2019) 451-458.

29. C. Li, L. Du, Y. Ren, X. Liu, Q. Jiao, D. Cui, M. Wen, C. Wang, G. Wei, Y. Wang, A. Ji, Q. Wang, SKP2 promotes breast cancer tumorigenesis and radiation tolerance through PDCD4 ubiquitination, Journal of experimental \& clinical cancer research : CR 38(1) (2019) 76.

30. C. Yang, J. Zhang, M. Ding, K. Xu, L. Li, L. Mao, J. Zheng, Ki67 targeted strategies for cancer therapy, Clinical \& translational oncology : official publication of the Federation of Spanish Oncology Societies and of the National Cancer Institute of Mexico 20(5) (2018) 570-575.

31. Z. Wang, H. Fukushima, H. Inuzuka, L. Wan, P. Liu, D. Gao, F.H. Sarkar, W. Wei, Skp2 is a promising therapeutic target in breast cancer, Frontiers in oncology 1(57) (2012).

32. Z. Wang, H. Inuzuka, J. Zhong, P. Liu, F.H. Sarkar, Y. Sun, W. Wei, Identification of acetylationdependent regulatory mechanisms that govern the oncogenic functions of Skp2, Oncotarget 3(11) (2012) 1294-300.

33. H. Zhu, L.L. Chang, F.J. Yan, Y. Hu, C.M. Zeng, T.Y. Zhou, T. Yuan, M.D. Ying, J. Cao, Q.J. He, B. Yang, AKR1C1 Activates STAT3 to Promote the Metastasis of Non-Small Cell Lung Cancer, Theranostics 8(3) (2018) 676-692.

34. N.M. Aiello, Y. Kang, Context-dependent EMT programs in cancer metastasis, The Journal of experimental medicine 216(5) (2019) 1016-1026.

35. W. Lu, Y. Kang, Epithelial-Mesenchymal Plasticity in Cancer Progression and Metastasis, Developmental cell 49(3) (2019) 361-374.

36. A. Nieszporek, K. Skrzypek, G. Adamek, M. Majka, Molecular mechanisms of epithelial to mesenchymal transition in tumor metastasis, Acta biochimica Polonica 66(4) (2019) 509-520.

37. I. Pastushenko, C. Blanpain, EMT Transition States during Tumor Progression and Metastasis, Trends in cell biology 29(3) (2019) 212-226.

38. M.V. Recouvreux, M.R. Moldenhauer, K.M.O. Galenkamp, M. Jung, B. James, Y. Zhang, A. Lowy, A. Bagchi, C. Commisso, Glutamine depletion regulates Slug to promote EMT and metastasis in pancreatic cancer, The Journal of experimental medicine 217(9) (2020).

39. B.N. Smith, N.A. Bhowmick, Role of EMT in Metastasis and Therapy Resistance, Journal of clinical medicine 5(2) (2016).

40. Y.W. Chang, Y.F. Zhao, Y.L. Cao, X.F. Gu, Z.Q. Li, S.Q. Wang, J.H. Miao, H.S. Zhan, Liver X receptor a inhibits osteosarcoma cell proliferation through up-regulation of Fox01, Cell Physiol Biochem 32(1) (2013) 180-6.

41. Z.C. Li, L.M. Zhang, H.B. Wang, J.X. Ma, J.Z. Sun, Curcumin inhibits lung cancer progression and metastasis through induction of FOX01, Tumour Biol 35(1) (2014) 111-6.

42. D. McHugh, J. Gil, Senescence and aging: Causes, consequences, and therapeutic avenues, J Cell Biol 217(1) (2018) 65-77.

43. T. von Zglinicki, G. Saretzki, J. Ladhoff, F. d'Adda di Fagagna, S.P. Jackson, Human cell senescence as a DNA damage response, Mechanisms of ageing and development 126(1) (2005) 111-7. 
44. C. Wang, D. Jurk, M. Maddick, G. Nelson, C. Martin-Ruiz, T. von Zglinicki, DNA damage response and cellular senescence in tissues of aging mice, Aging cell 8(3) (2009) 311-23.

45. N. Alessio, D. Aprile, T. Squillaro, G. Di Bernardo, M. Finicelli, M.A. Melone, G. Peluso, U. Galderisi, The senescence-associated secretory phenotype (SASP) from mesenchymal stromal cells impairs growth of immortalized prostate cells but has no effect on metastatic prostatic cancer cells, Aging (Albany NY) 11(15) (2019) 5817-5828.

46. W.Y. Kim, N.E. Sharpless, The regulation of INK4/ARF in cancer and aging, Cell 127(2) (2006) 265-75.

47. A. Calcinotto, J. Kohli, E. Zagato, L. Pellegrini, M. Demaria, A. Alimonti, Cellular Senescence: Aging, Cancer, and Injury, Physiological reviews 99(2) (2019) 1047-1078.

48. P.J. Barnes, J. Baker, L.E. Donnelly, Cellular Senescence as a Mechanism and Target in Chronic Lung Diseases, American journal of respiratory and critical care medicine 200(5) (2019) 556-564.

49. Y. Liu, O.E. Hawkins, Y. Su, A.E. Vilgelm, T. Sobolik, Y.M. Thu, S. Kantrow, R.C. Splittgerber, S. Short, K.I. Amiri, J.A. Ecsedy, J.A. Sosman, M.C. Kelley, A. Richmond, Targeting aurora kinases limits tumour growth through DNA damage-mediated senescence and blockade of NF-KB impairs this drug-induced senescence, EMBO Mol Med 5(1) (2013) 149-66.

50. S.G. Rao, J.G. Jackson, SASP: Tumor Suppressor or Promoter? Yes!, Trends Cancer 2(11) (2016) 676687.

51. J.M. van Deursen, Senolytic therapies for healthy longevity, Science 364(6441) (2019) 636-637.

52. S. Short, E. Fielder, S. Miwa, T. von Zglinicki, Senolytics and senostatics as adjuvant tumour therapy, EBioMedicine 41 (2019) 683-692.

53. L. Samaraweera, A. Adomako, A. Rodriguez-Gabin, H.M. McDaid, A Novel Indication for Panobinostat as a Senolytic Drug in NSCLC and HNSCC, Sci Rep 7(1) (2017) 1900.

54. Y. Ovadya, V. Krizhanovsky, Strategies targeting cellular senescence, J Clin Invest 128(4) (2018) 1247-1254.

\section{Figures}


B

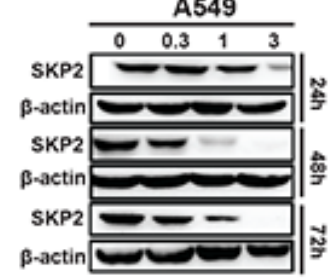

D

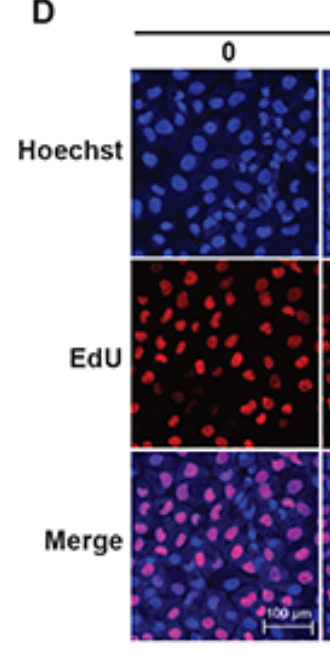

E

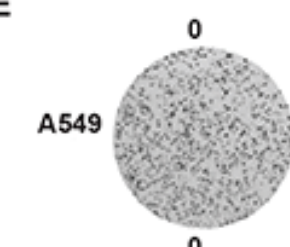

0

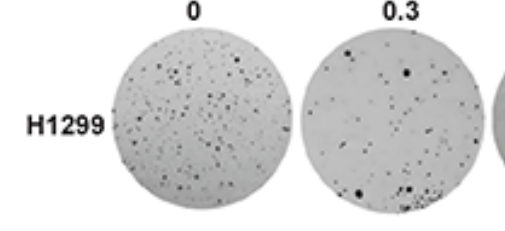

0.3

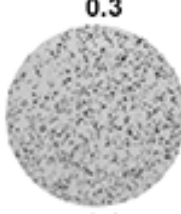

3

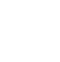

C

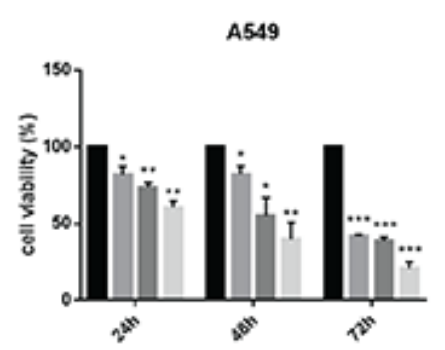

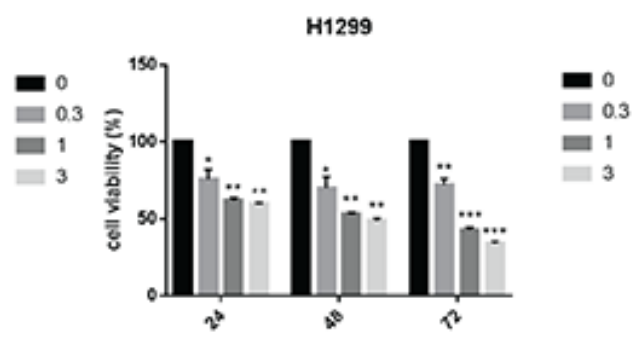

H1299

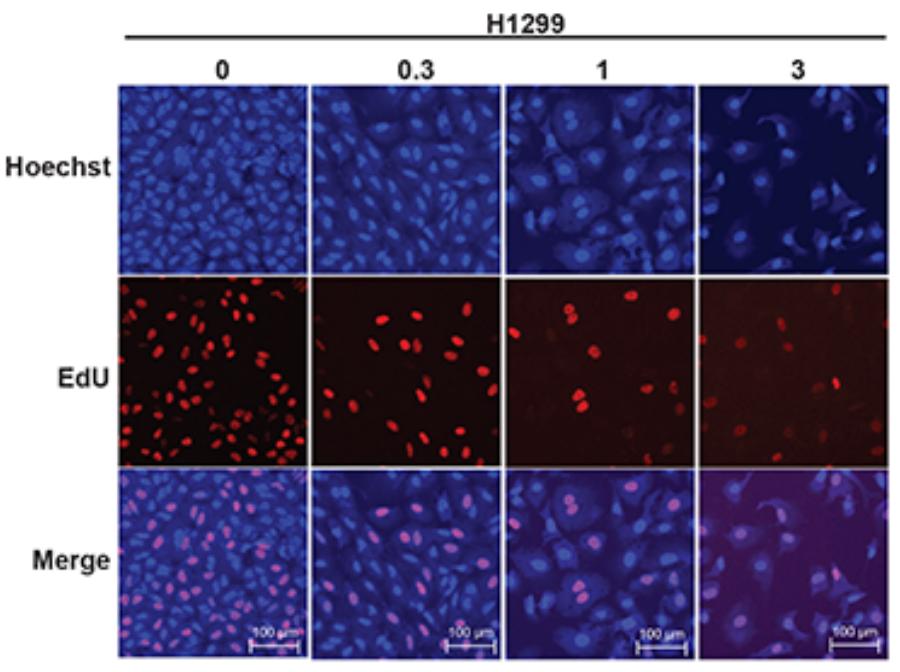

A549

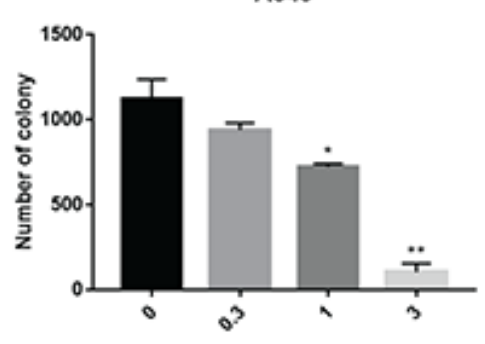

H1299

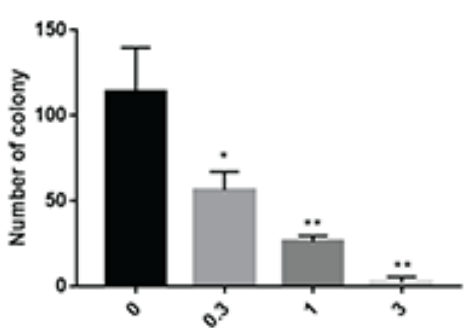

Figure 1

AAA-237 inhibited proliferation of A549 and H1299 cells in a dose- and time-dependent manner. A. The structure of AAA-237. B. AAA-237 inhibited the expression of Skp2 in a dose and time-depend manner. C. CCK8 assay showed that AAA-237 inhibits proliferation of A549 and H1299 cells. D. EdU-DNA synthesis assay showed that AAA-237 inhibited DNA synthesis in A549 and H1299 cells. Scale bar $=100 \mu \mathrm{m}$. E. AAA-237 inhibited colony formation of the A549 and H1299 cells. The experiments were performed in triplicate, and the data are presented as mean $\pm S D$, ${ }^{*}<0.05$, $* * P<0.01$, ${ }^{\star \star *} P<0.005$ vs. control group. 
A
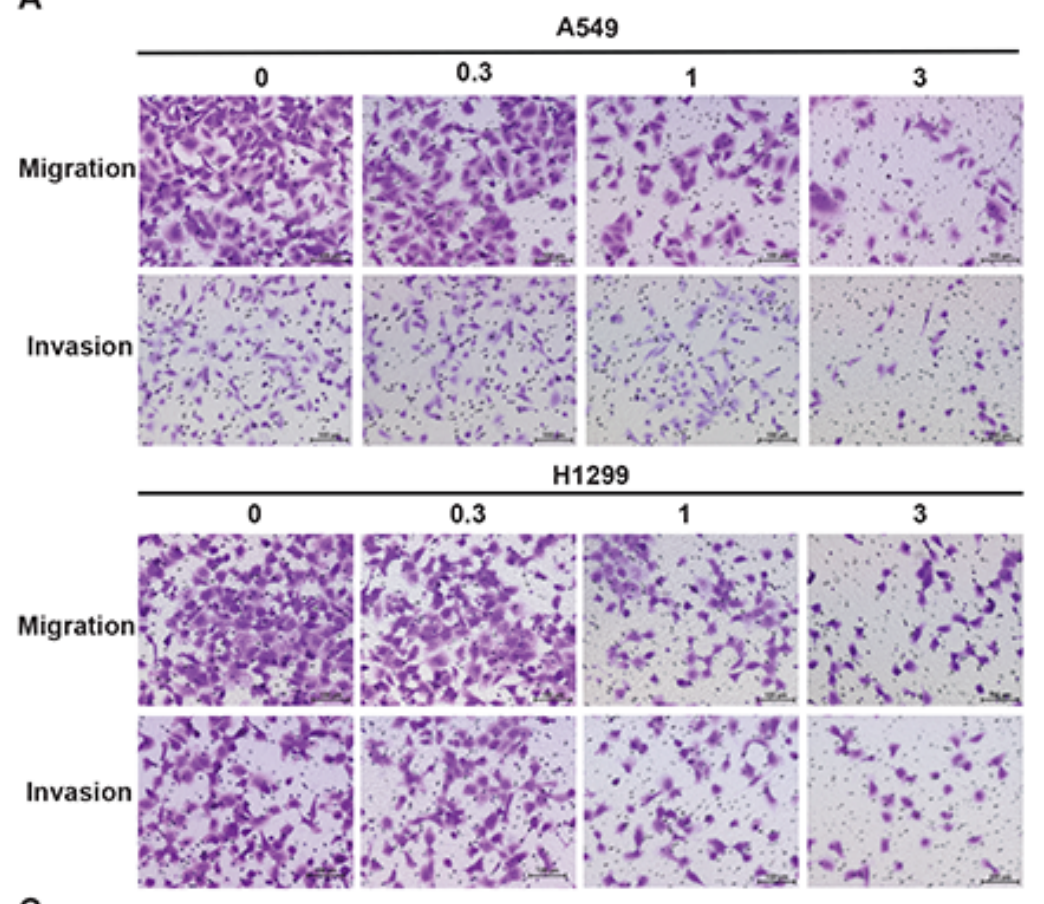

C

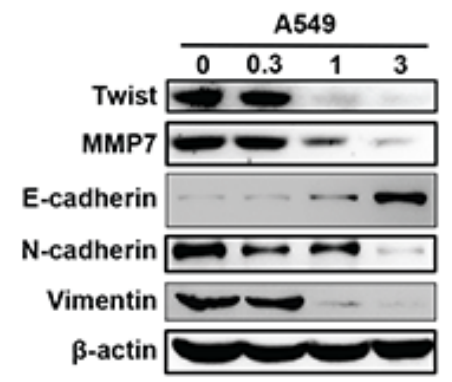

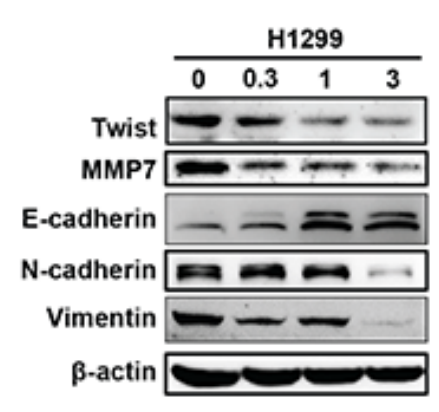

B
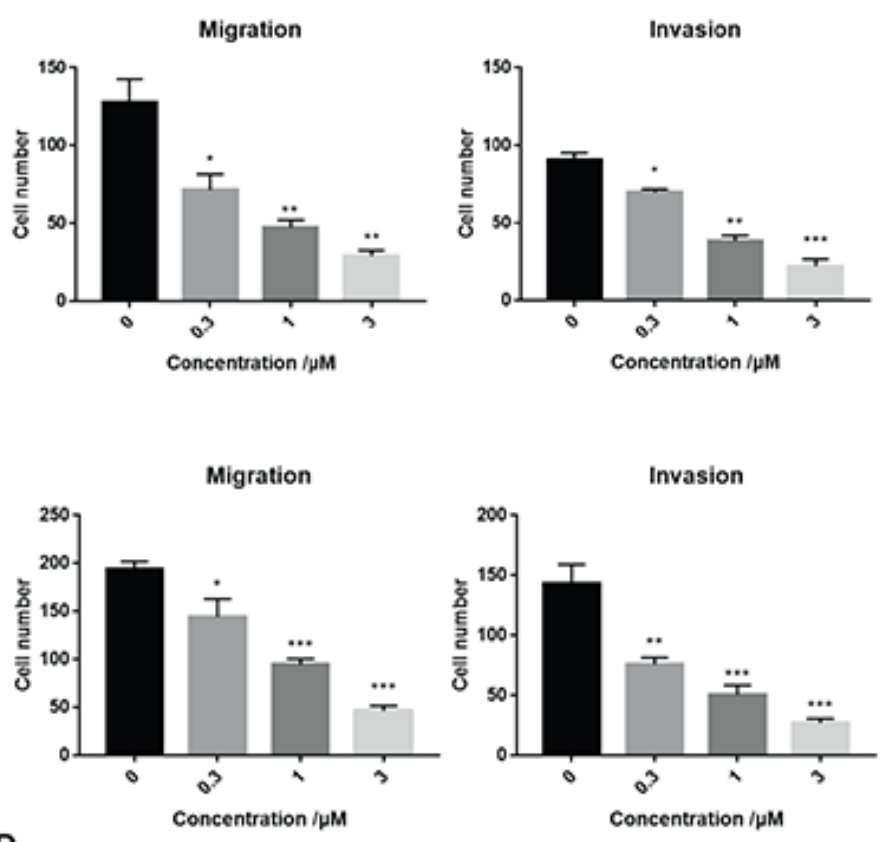

D

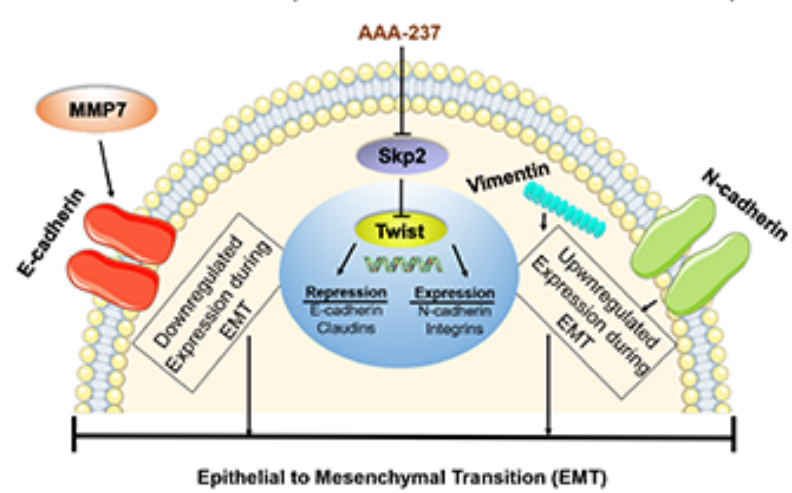

Figure 2

AAA-237 inhibited migration and invasion of A549 and H1299 cells. A-B. Transwell assays showed that AAA-237 inhibited migration and invasion of A549 and H1299 cells. Scale bar $=100 \mu \mathrm{m}$. C. AAA-237 regulated EMT by Skp2/Twist axis. The expression of twist, MMP7, N-cadherin and Vimentin was reduced whereas the expression of E-cadherin was increased. D. Schematic model for the mechanism of AAA-237 in migration and invasion. The experiments were performed in triplicate, and the data are presented as mean $\pm S D, * P<0.05$, ${ }^{\star *} P<0.01$, ${ }^{\star *} * \mathrm{P}<0.005$ vs. control group. 
A

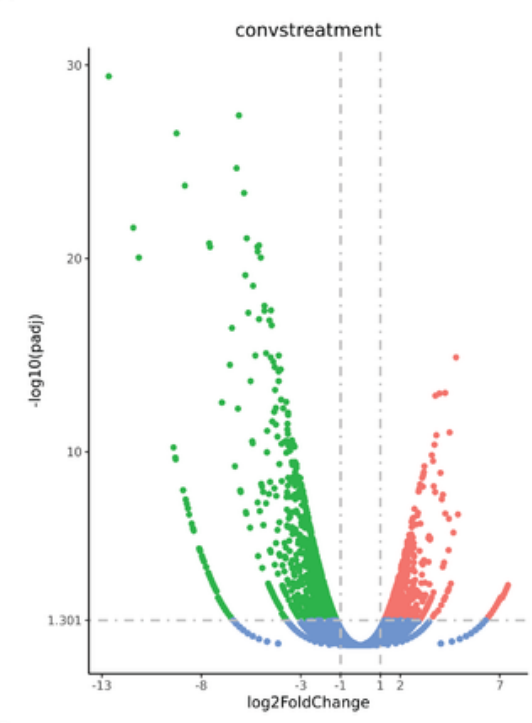

C

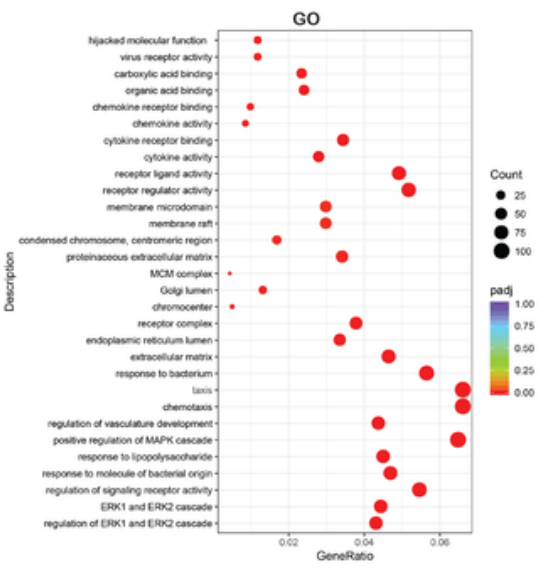

B

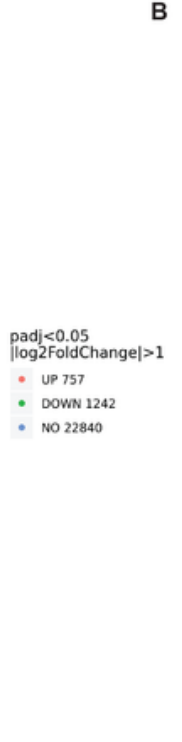

D

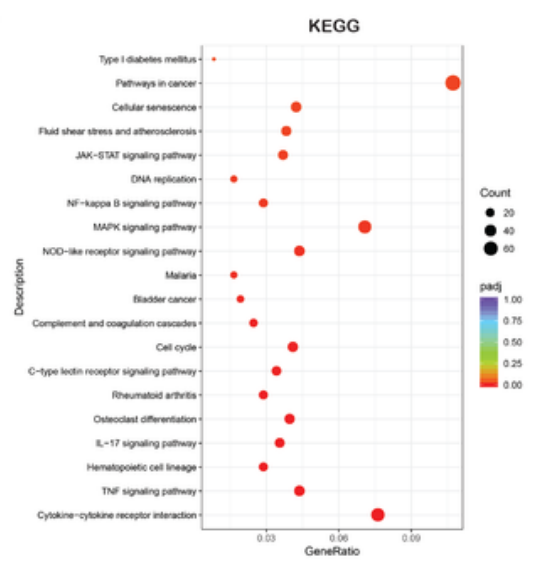

Pathway in cancer PTGS2 BIRC3 PGF RUNX1 JUN PMAIPI PML RPSGKAS RET STAT5A TGFA (IK3CD TNESF9 FUS WNI4 NFKB1) WNT7A TRAF1 MAPK13 CXCR4 NRX3-1 SLC2A)

PLK3 PTIG1 BUB1B CDKN2D CCND3 BUIB1 CDC45 MCM3 PLN1 CDC25C MAD2L CDC20 CGIVB1 DBF4 CDC7 ELr2 CDC25A CCNA2 CCNG2 CunB2 CDKN2CGADD45 ESPL1) SKP2 CDKN1A RBL1 Cell cycle

\section{Figure 3}

Differential expression and enrichment analysis of the A549 cells treated with AAA-237. A. Volcano plot of differential expression results (upregulated genes are in red, downregulated genes are in green, nonregulated genes are in blue ( $|\log 2 \mathrm{FC}| \geq 1$ and $P$ value $\leq 0.05)$. B. Heatmap of differentially expressed genes. C. GO enrichment analysis of differentially expressed genes. D. KEGG pathway analysis of 
differentially expressed genes. E. Protein-protein interactions. The network was visualized using Cytoscape 3.6.0.

A
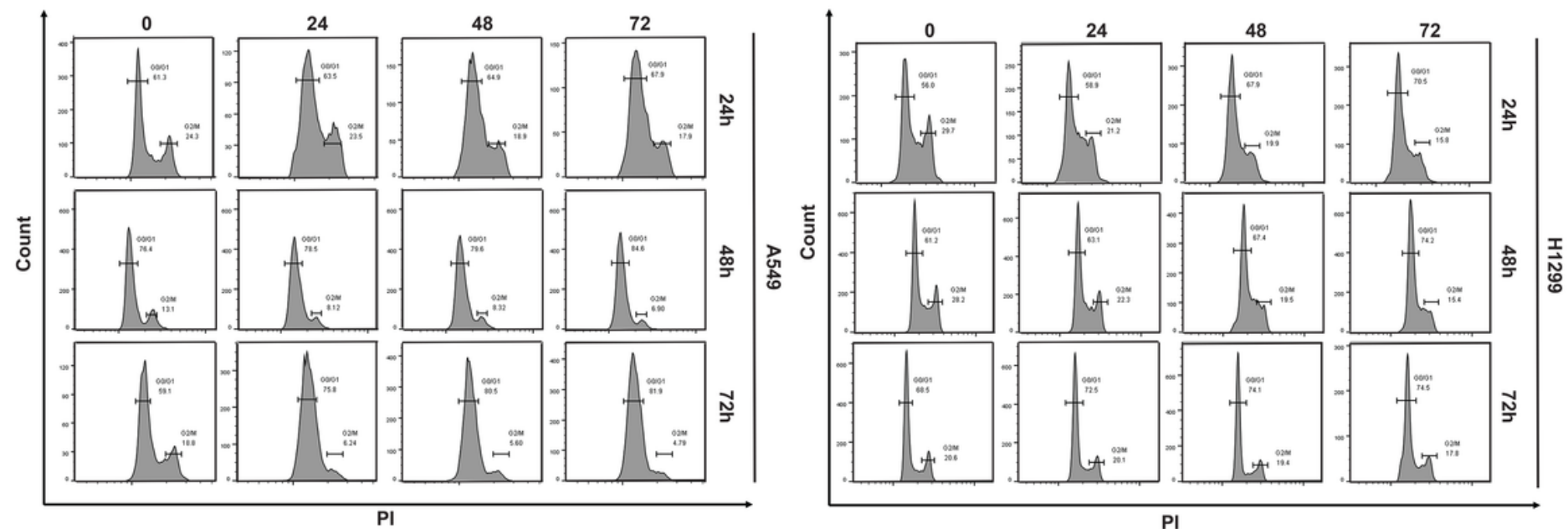

B
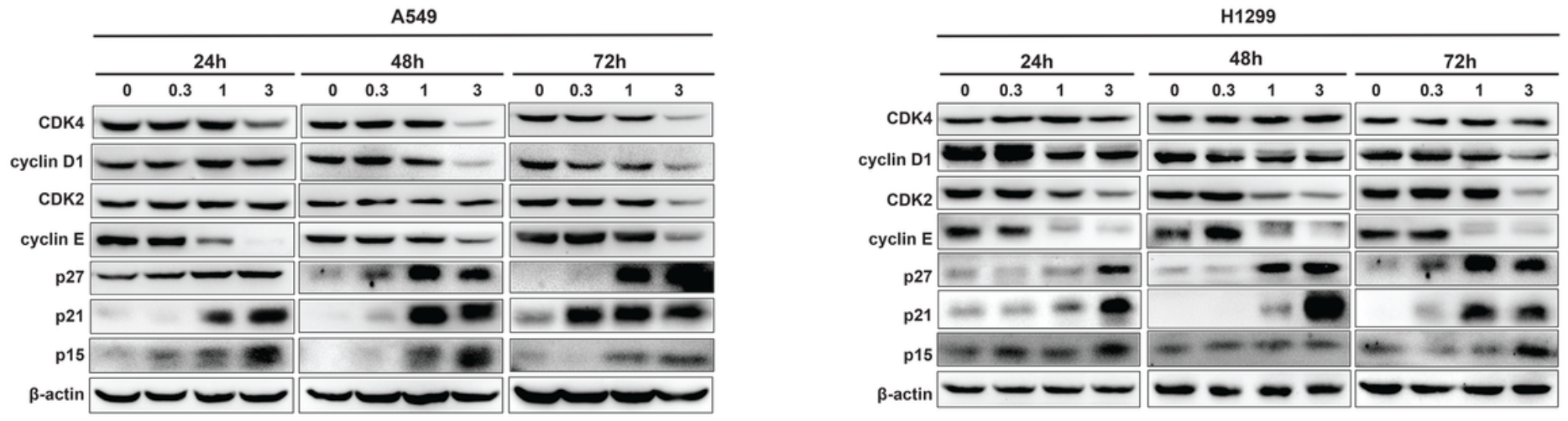

C
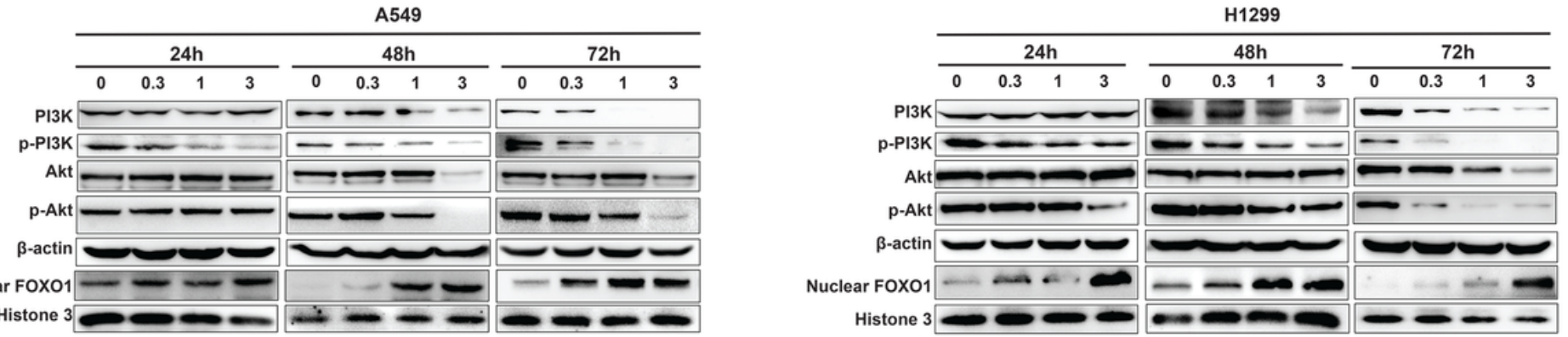

\section{Figure 4}

AAA-237 arrested cell cycle at G0/G1 in A549 and H1299 cells. A. Flow cytometric analysis showed that AAA-237 arrested the cell cycle at G0/G1 phase in A549 and H1299 cells. B. Western blotting results showed that AAA-237 regulated the expression of cell cycle-related proteins A549 and H1299 cells. C. Western blotting results showed that AAA-237 regulated PI3K/Akt-FOX01 signaling pathways. The experiments were performed in triplicate. 
A

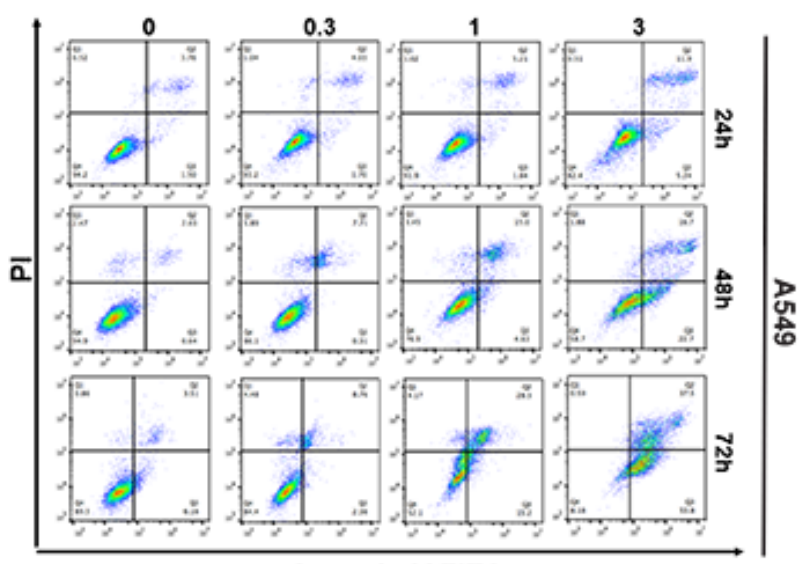

Annexin-V-FITC

B

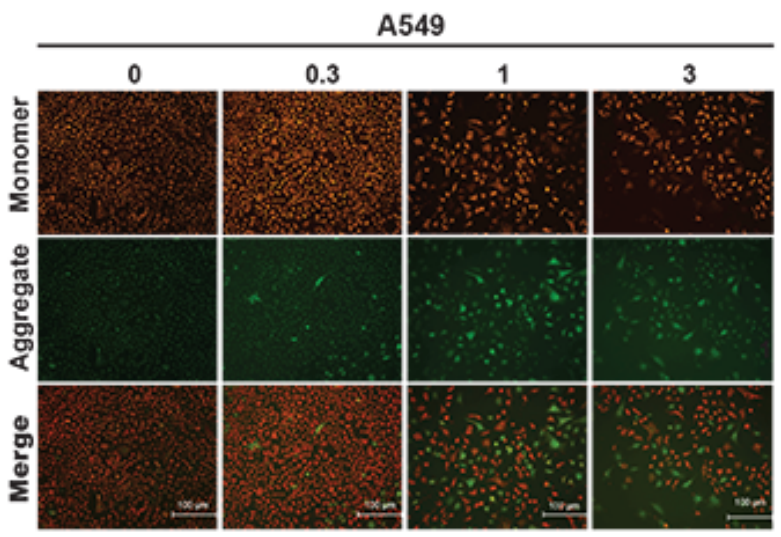

C

D
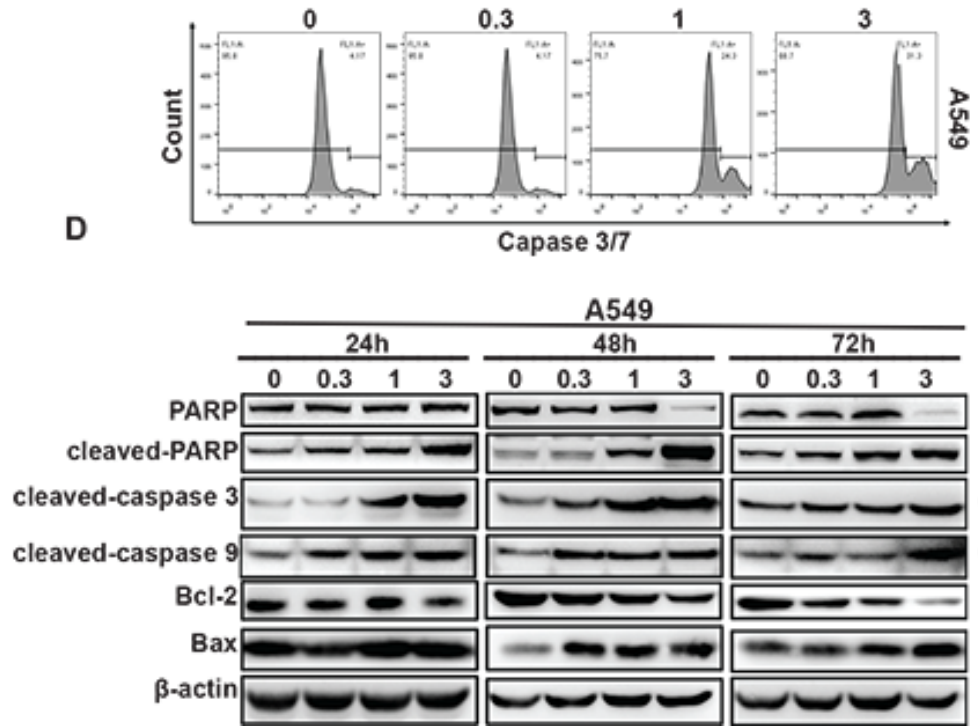

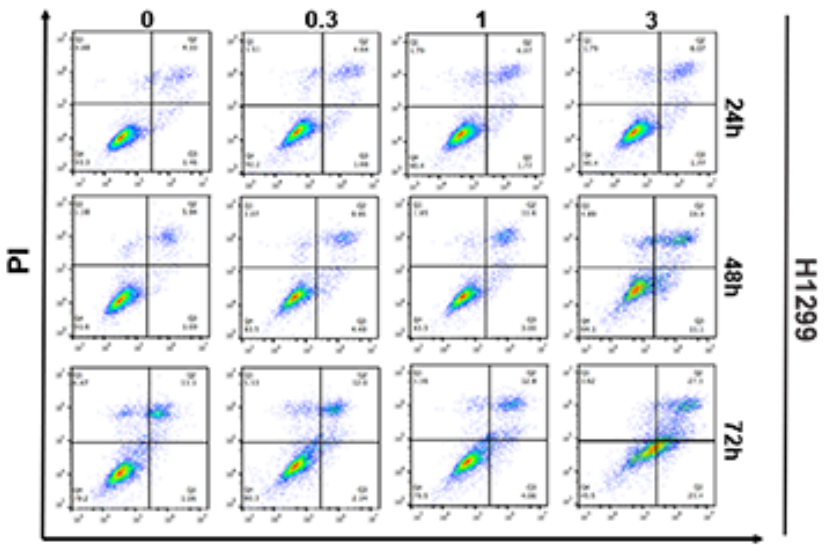

Annexin-V-FITC
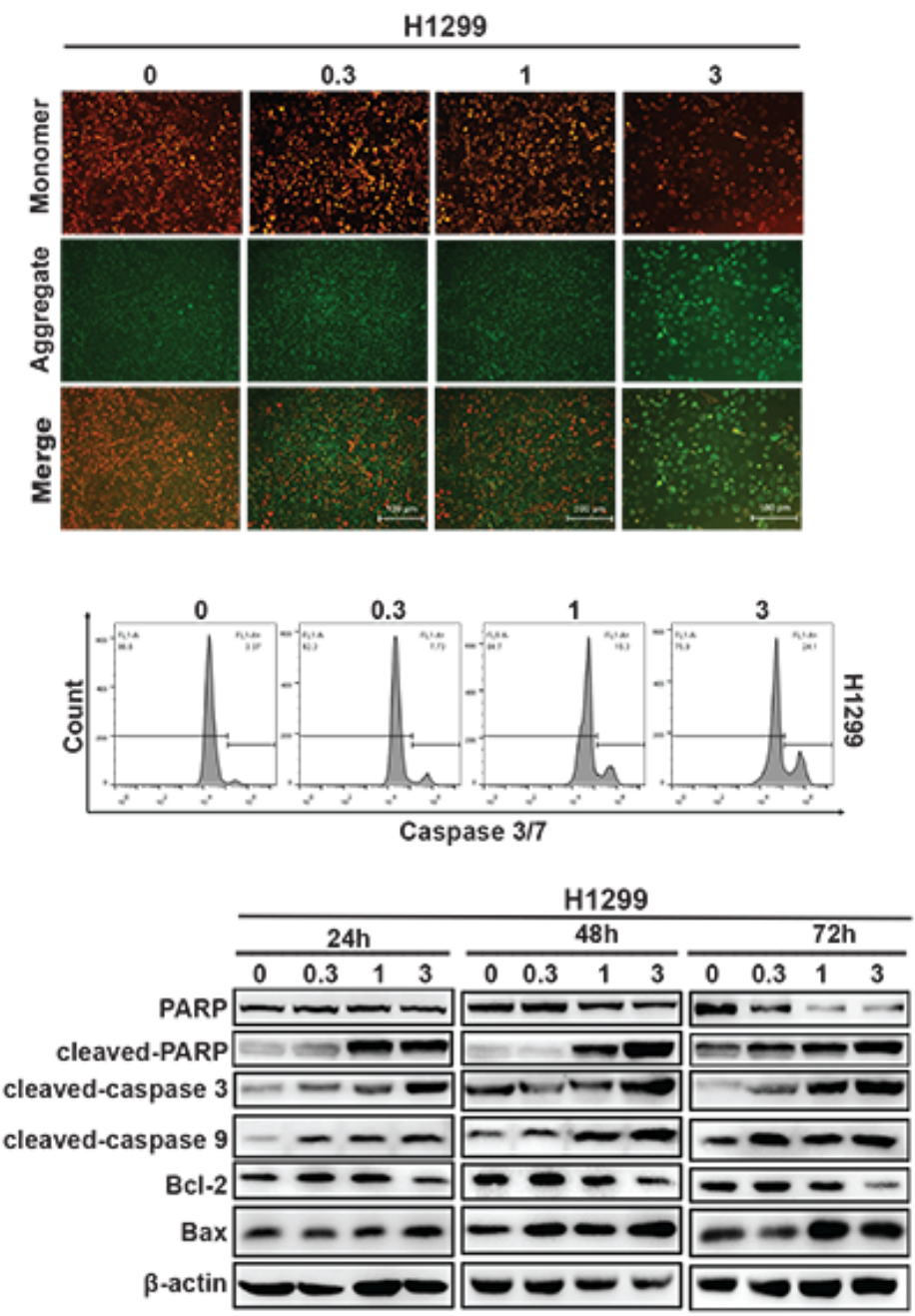

\section{Figure 5}

AAA-237 induced apoptosis of A549 and H1299 cells in a mitochondria-dependent manner. A. Flow cytometric analysis showed that AAA-237 increased the apoptosis rate of the A549 and H1299 cells. B. JC-1 staining assays showed that AAA-237 reduced the mitochondrial membrane potential of the A549 and $\mathrm{H} 1299$ cells. Scale bar $=100 \mu \mathrm{m}$. C. Flow cytometric analysis of caspase 3/7 activity showed that AAA-237 increased caspase 3/7 activity of the A549 and H1299 cells. D. Western blotting results showed 
that AAA-237 regulated the expression of apoptosis-related proteins in A549 and H1299 cells. The experiments were performed in triplicate.

A
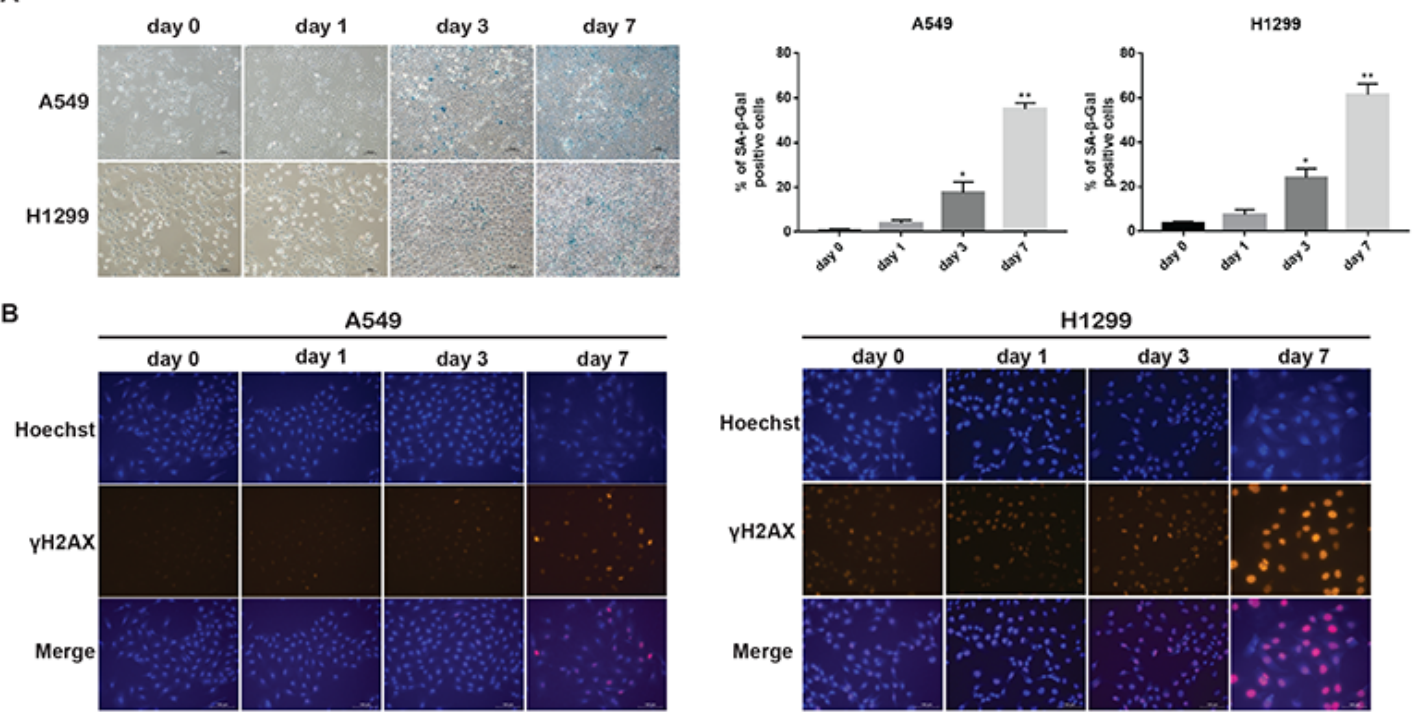

D
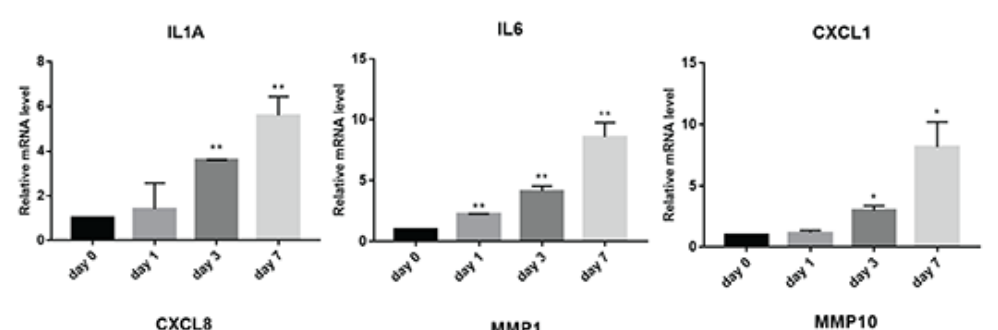

C
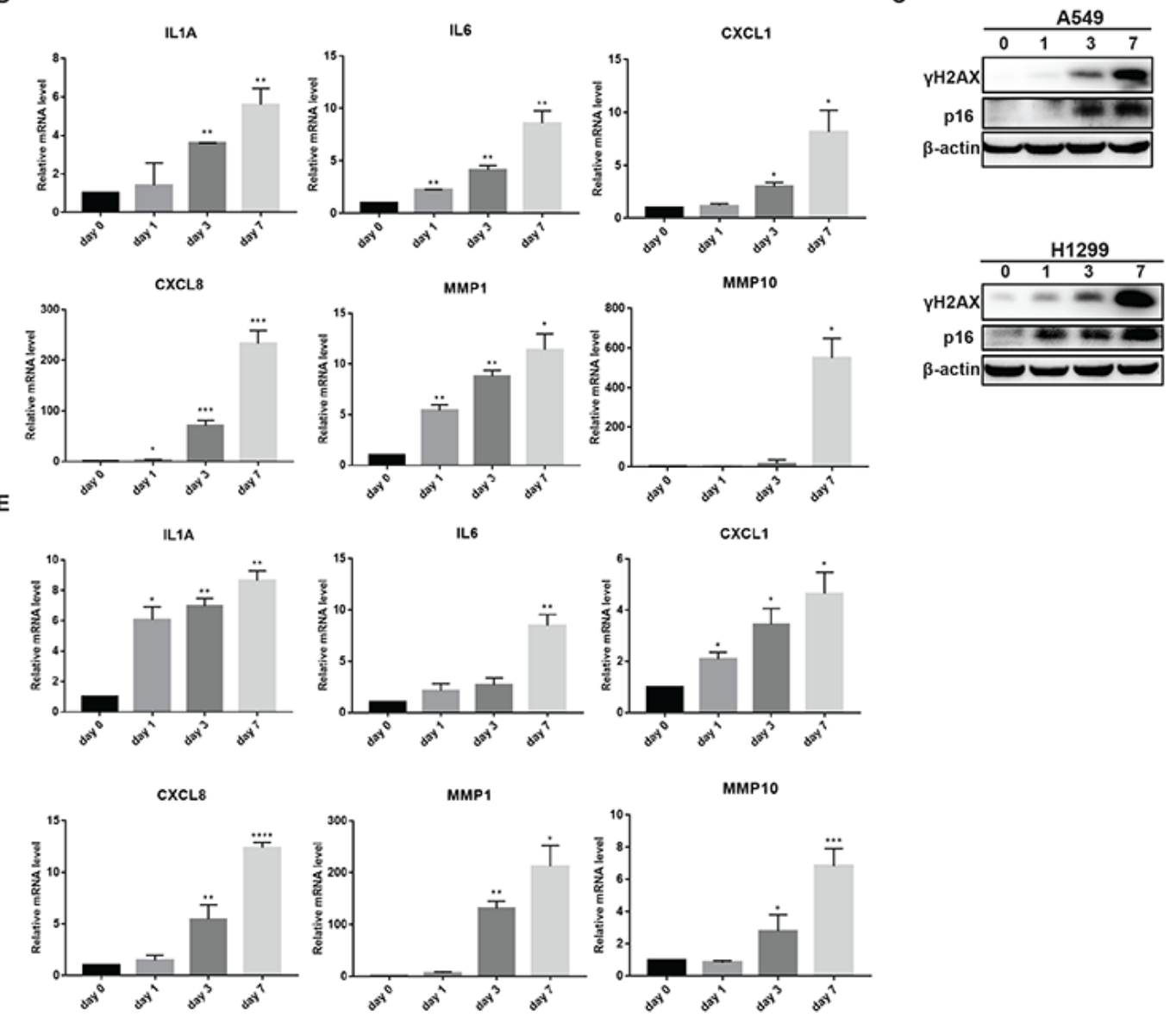

\section{Figure 6}

AAA-237 induced senescence of A549 and H1299 cells. A. SA- $\beta-G a l$ assays showed that treatment of low concentration AAA-237 for long-term induced senescence of A549 and H1299 cells. Scale bar $=100 \mu \mathrm{m}$. B. Immunofluroescence assays showed that treatment of low concentration AAA-237 for long-term 
induced the expression of senescence marker $\mathrm{YH} 2 \mathrm{~A}$.X in the A549 and H1299 cells. Scale bar $=100 \mu \mathrm{m}$. C. Western blotting results showed that treatment of low concentration AAA-237 for long-term increased the expression of senescence markers yH2A.X and p16 in A549 and H1299 cells. D. Real-time qPCR analysis showed that treatment of low concentration AAA-237 for long-term increased the expression of SASP factors IL1A, IL6, CXCL1, CXCL8, MMP1 and MMP10 in A549 cells. E. Real-time qPCR analysis showed that treatment of low concentration AAA-237 for long-term increased the expression of SASP factors IL1A, IL6, CXCL1, CXCL8, MMP1 and MMP10 in H1299 cells. The experiments were performed in triplicate, and the data are presented as mean $\pm S D$, ${ }^{*} P<0.05$, $* * P<0.01$, $* * * P<0.005$ vs. control group.

A

A549
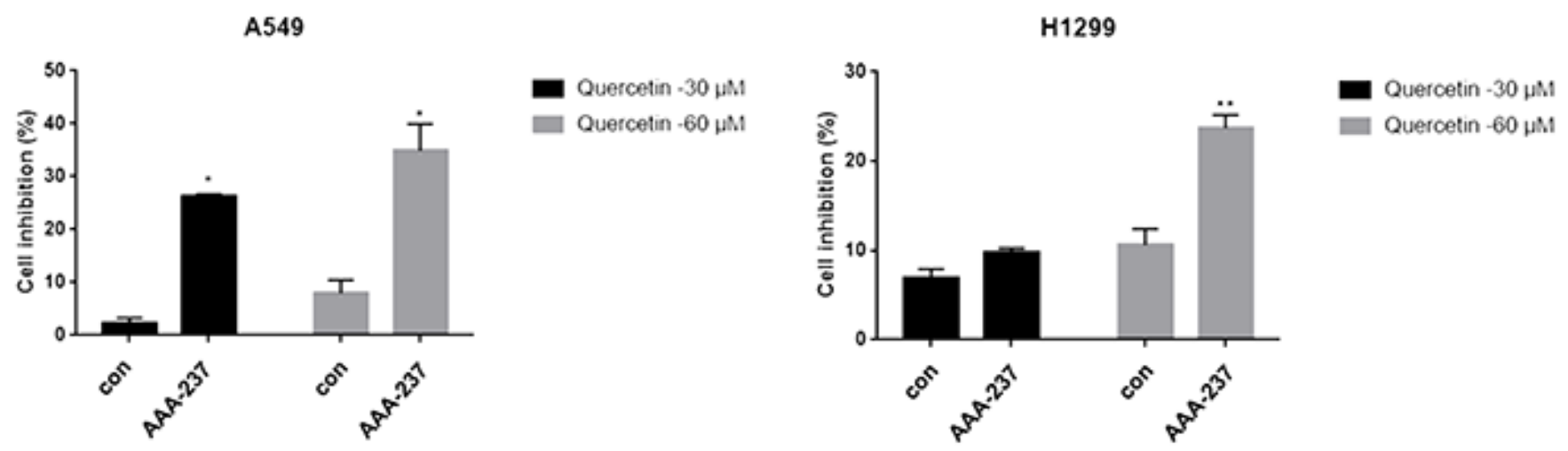

B

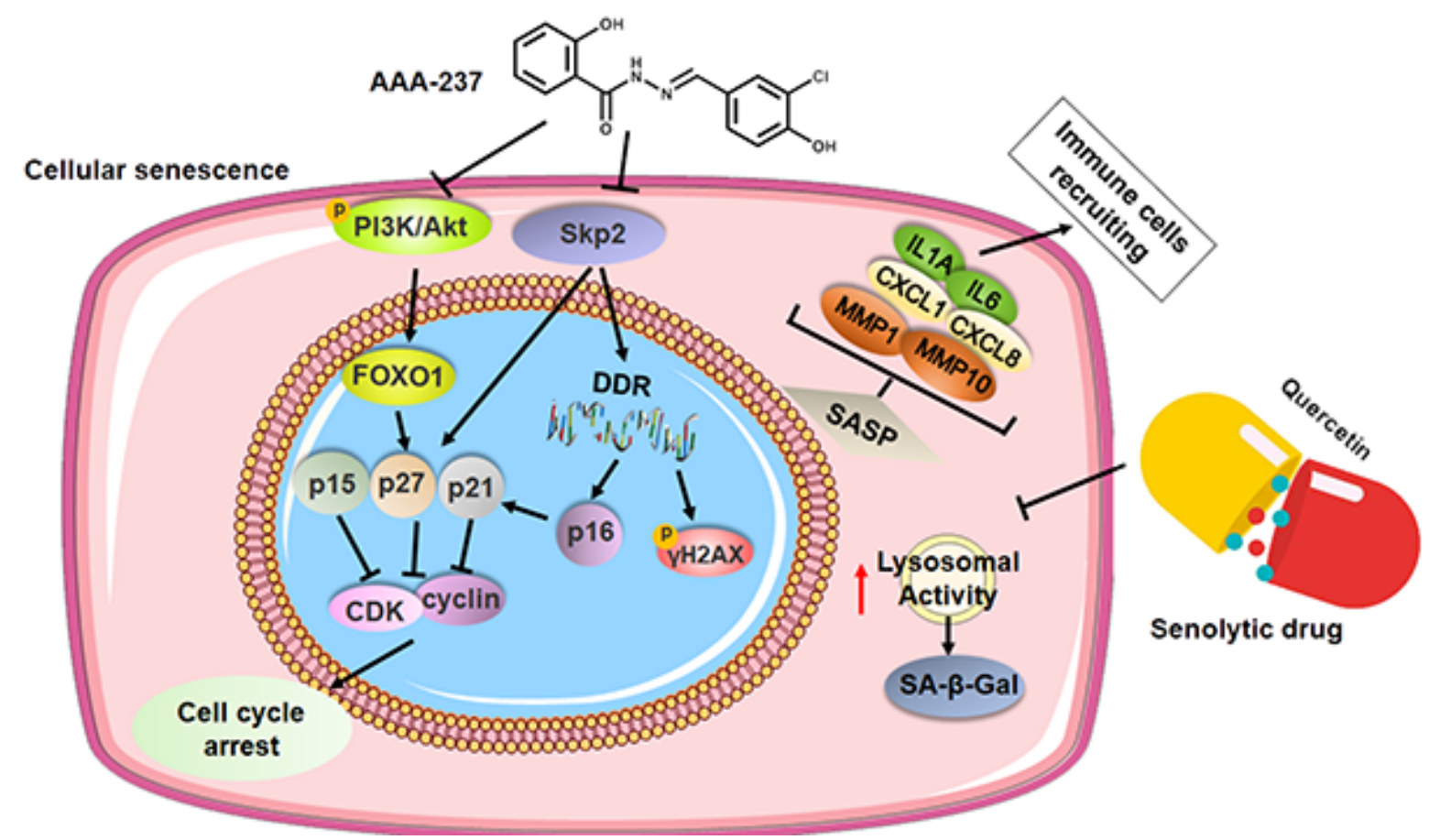

Figure 7

Effects of senolytic drug quercetin on AAA-237-induced senescent A549 and H1299 cells. A. CCK8 assay showed that quercetin reduced the viability of AAA-237-treated A549 and H1299 cells. B. Schematic model for the mechanism of AAA-237 induced senescence in NSCLC cell. The experiments were performed in triplicate, and the data are presented as mean $\pm S D,{ }^{*} P<0.05,{ }^{*} P<0.01$ vs. control group. 


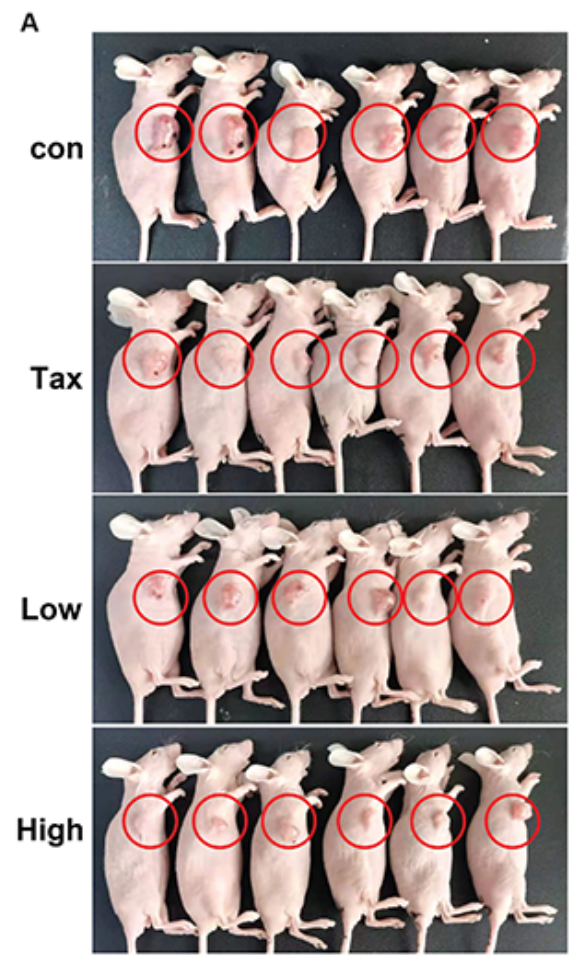

C

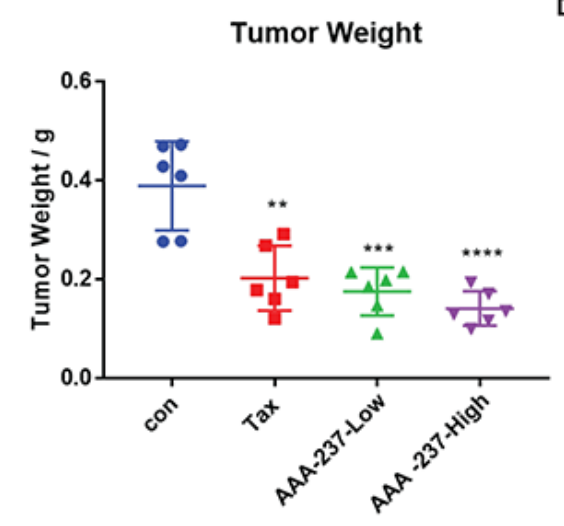

Organ index

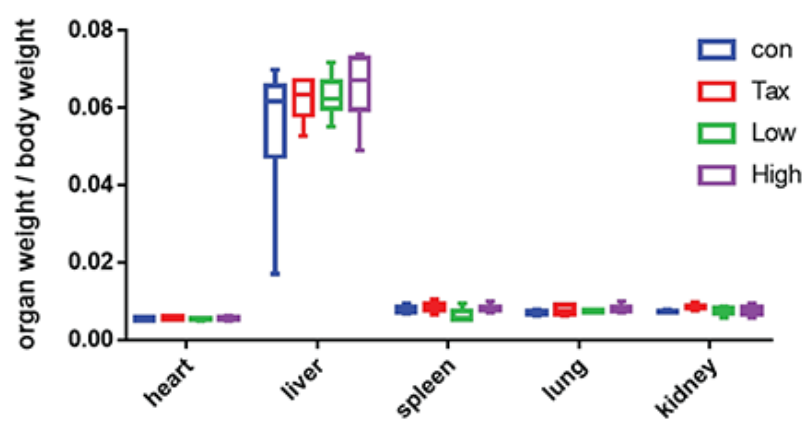

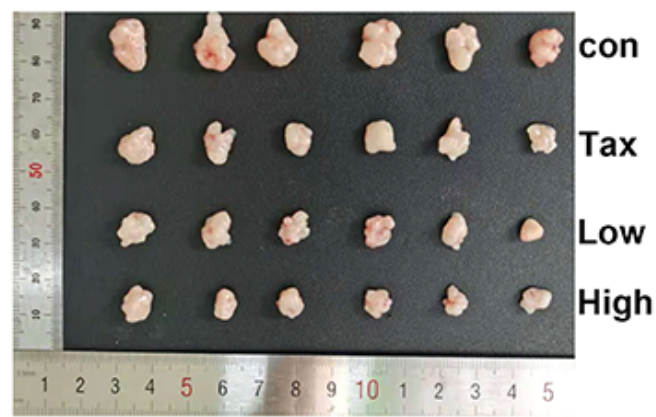

B

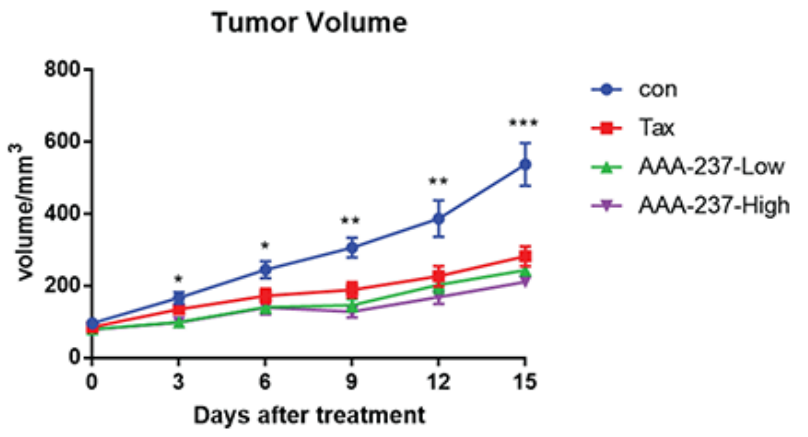

D

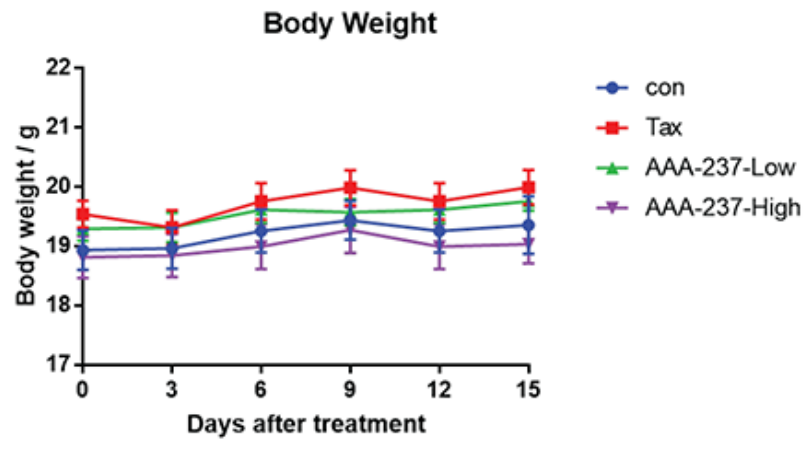

\section{Figure 8}

AAA-237 suppresses growth of xenograft tumors. A. Image of xenograft tumors. B. AAA-237 reduced tumor volume. C. AAA-237 reduced tumor weight. D. Body weight during treatment of AAA-237. E. Relative organ weights after treatment of AAA-237, $n=6$. The data were presented as mean $\pm S D, \star \star P<0.01, \star \star \star P<$ $0.005, \star \star \star \star P<0.001$ vs. control group. 
A

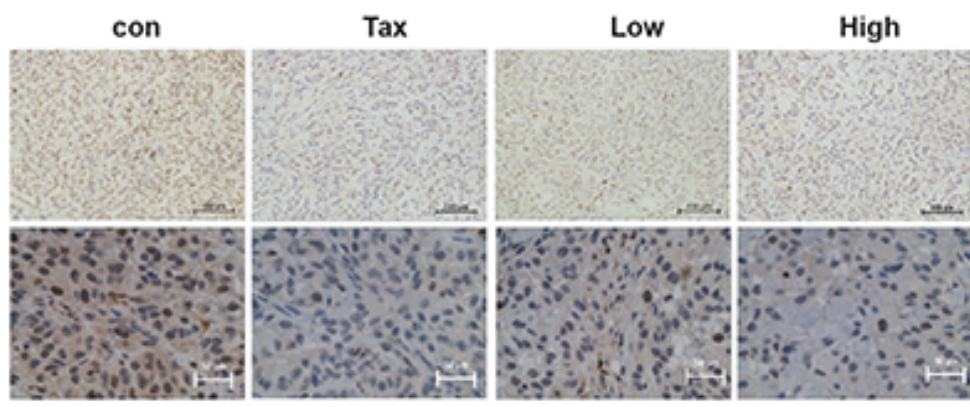

B

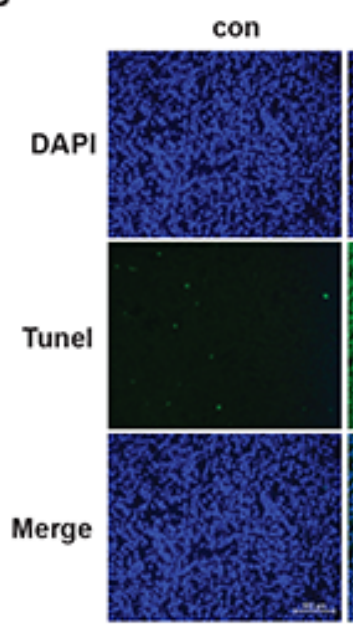

$\operatorname{Tax}$

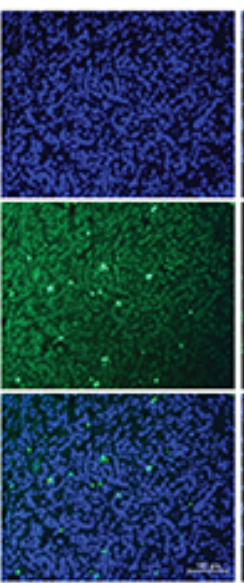

Low

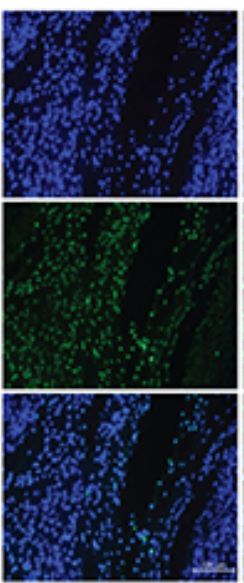

High

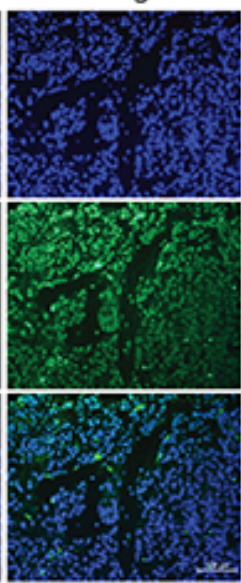

C

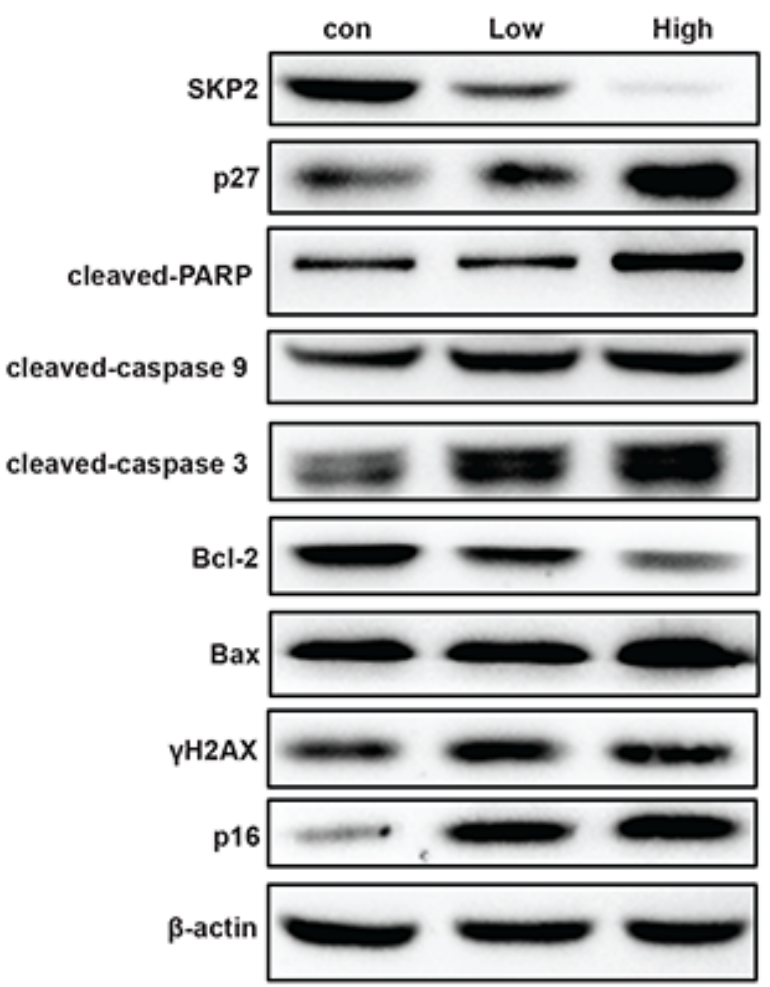

Figure 9

AAA-237 suppresses growth of xenograft tumors by inducing apoptosis and senescence in vivo. A. Immunohistochemistry analysis showed that AAA-237 reduced the expression of Ki67 in tumor tissue. Scale bar $=100 \mu \mathrm{m}$. B. Tunel stain assay showed that AAA-237 induced apoptosis of cells in tumor tissue. Scale bar $=100 \mu \mathrm{m}$. C. Western blotting results showed that AAA-237 reduced the expression of Skp2 and Bcl-2 and increased the expression of p27, the cleaving of caspase 3, caspase 9, PARP and Bax. The expression of senescence-related proteins $\mathrm{yH} 2 \mathrm{~A} . \mathrm{X}$ and $\mathrm{p} 16$ is also up-regulated. The experiments were performed in triplicate.

\section{Supplementary Files}

This is a list of supplementary files associated with this preprint. Click to download.

- Graphicabstract.tif

- supplementarymeterial.docx 